\title{
Der Lehrer und sein Schüler. Die Korrespondenz zwischen Lorenz Heister und Christoph Jacob Trew
}

Marion Maria Ruisinger und Thomas Schnalke

\section{Summary}

Letters were the central medium of communication in the medical scientific community of the 18 th century. Professional as well as personal relationships were established among the various correspondents. These relationships constituted the smallest units of communication which contributed to the regional and international scientific network of the Republic of Letters. A correspondence that grew out of a trusted teacher-student relationship could gain an especially intense character both intellectually and personally. This contribution offers an analysis of an example of just such a correspondence. Lorenz Heister (1683-1758), medical professor at the universities of Altdorf and Helmstedt, and his disciple, Christoph Jacob Trew (1695-1769), who became a renowned physician and natural scientist in Nuremberg, communicated in letters to one another over a span of almost forty years. Their correspondence started as a rather asymmetrical dialogue. Over time, however, Heister and Trew came to meet as equals in almost every field of their competence. Their letters reveal a broad spectrum of scientific, organisational, professional, medical and personal issues which formed the basis of a stable and lasting learned correspondence in the age of Enlightenment.

Keywords: medical correspondence (18th century); Lorenz Heister (16831758); Christoph Jacob Trew (1695-1769); medical communication (18th century); scientific networks (18th century)

Dr. Marion Maria Ruisinger, Institut für Geschichte und Ethik der Medizin, Glückstrasse 10, D-91054 Erlangen (marion.ruisinger@gesch.med.uni-erlangen.de).

Prof. Dr. Thomas Schnalke, Berliner Medizinhistorisches Museum der Charité, Schumannstrasse 20/21, D-10117 Berlin (thomas.schnalke@charite.de). 


\section{Zusammenfassung}

Briefe bildeten das zentrale Kommunikationsmedium der medizinischen Gelehrtenwelt des 18. Jahrhunderts. Zwischen den Korrespondenten konstituierten sich unterschiedliche beruflich-persönliche Beziehungsverhältnisse als kleinste Kommunikationseinheiten, die sich zu dem überregional und letztlich international angelegten wissenschaftlichen Netzwerk der Gelehrtenrepublik zusammenfügten. - Eine fachlich wie persönlich besonders enge Prägung erfuhren Briefpartnerschaften, die aus einem vertrauten LehrerSchüler-Verhältnis erwuchsen. Der Beitrag liefert eine Analyse der vier Jahrzehnte umfassenden Korrespondenz zwischen dem in Altdorf und Helmstedt lehrenden Medizinprofessor Lorenz Heister (1683-1758) und seinem Altdorfer Schüler, dem später in Nürnberg praktizierenden Arzt und Naturforscher Christoph Jacob Trew (1695-1769). Das anfänglich asymmetrische Verhältnis der Briefpartner entwickelte sich im Laufe der Zeit zu einer Kommunikation unter nahezu gleichrangigen Gelehrten. Dabei tritt ein breites Geflecht an wissenschaftlichen, wissenschaftsorganisatorischen, berufspolitischen, medizinischen und persönlichen Briefthemen zutage, die das Fundament einer tragfähigen und dauerhaften Gelehrtenkorrespondenz im Jahrhundert der Aufklärung ausmachten.

Briefe und Briefwechsel haben in den letzten Jahren als Quellenbestand der medizinhistorischen Forschung an Bedeutung gewonnen. Je nach Autor, Adressat und Gegenstand eröffnen sie Zugänge zu einem breiten Spektrum von Fragestellungen: Private Briefe aus der Feder medizinischer Laien bieten sich für Studien zur Alltags-, Patienten- oder Körpergeschichte $\mathrm{an}^{1}$; Konsiliarkorrespondenzen aus dem Nachlass praktisch tätiger Ärzte erlauben es, die Interaktion zwischen Arzt und Patient zu analysieren, und bieten zudem die Möglichkeit, durch Fokussierung auf die patientenseitigen Stimmen innerhalb der Briefwechsel die Wahrnehmungs-, Deutungs- und Handlungsmuster der Kranken zu beleuchten². Der schriftliche Austausch zwischen Ärzten, Naturforschern und anderen Gelehrten gewährt Einblicke

1 Exemplarisch seien die Arbeiten zu Liselotte von der Pfalz (Forster 1986), Zélie Martin (Taylor 1998) und Franz Xaver von Zach (Gosteli/Boschung/Brosche 1998) genannt, s.a. Barras/Dinges 2004.

2 Bearbeitet wurden bislang v.a. die ärztlichen Konsiliarkorrespondenzen von William Cullen (Risse 1974), Etienne-François Geoffroy (Brockliss 1994), Samuel Hahnemann (Gehrke 2000; Stolberg 1999; Meyer 1986), Lorenz Heister (Ruisinger 2003, 2001) und Auguste Tissot (Barras/Louis-Courvoisier 2001; Stolberg 2003, 1996). Vgl. auch Wild 2001. 


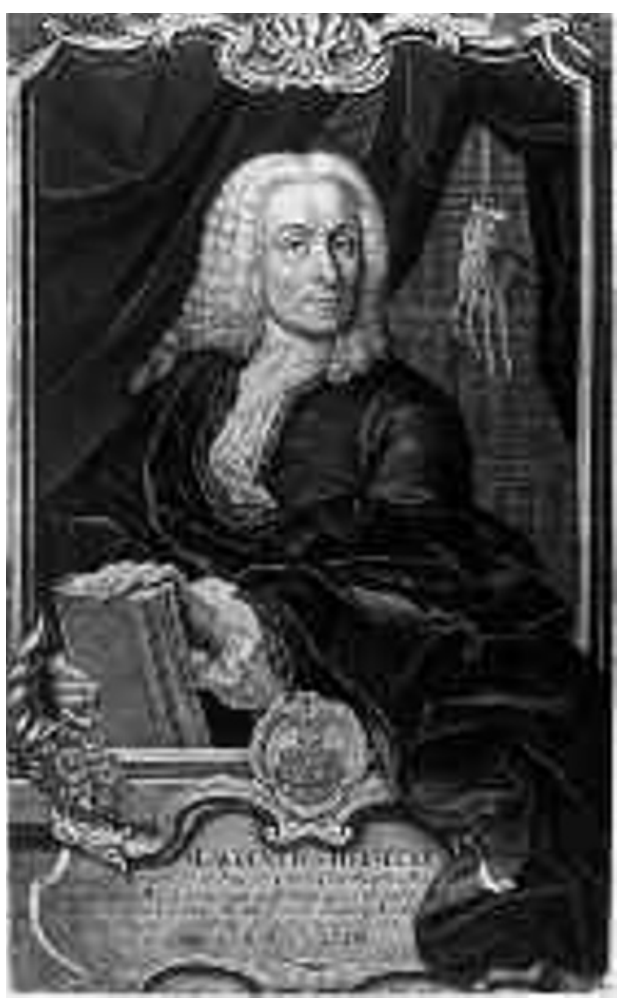

Abb.1. Lorenz Heister, Schabkunstblatt von Johann Jakob Haid (Bildarchiv Medizinhistorisches Institut Bern).

erfolgreicher Arzt, kenntnisreicher Botaniker und engagierter Wissenschaftsorganisator an Profil gewinnt.

Die Korrespondenz zwischen Heister und Trew wird heute in der Universitätsbibliothek Erlangen-Nürnberg aufbewahrt. 59 der insgesamt 77 Schriftstücke stammen von Heister, 18 Briefe und Briefentwürfe aus der Feder Trews. Das erste Schreiben verfasste Heister am 9. Februar 1717, den letzten Brief richtete am 20. Dezember 1757 gleichfalls Heister an Trew ${ }^{6}$. Der

3 Aus der Fülle der Arbeiten zu diesem Thema sei eine Auswahl jüngerer Werke genannt: Kempe 2000; Steinke 1999; Schnalke 1997 und 1994; Hörz 1996 sowie Rusnock 1996.

4 Eine ausführliche biographische Würdigung der Person Heisters unter Einbeziehung des reichen Quellenmaterials steht derzeit noch aus. Bislang liegen lediglich biographische Abrisse im Rahmen von Studien vor, die sich mit einem Teilaspekt des Heisterschen Werks beschäftigen, so zum Beispiel Geiger 1983; Korff 1975 und Schlipp 1910. - Die massgebliche Quelle für diese Bearbeitungen bilden die von Heister autorisierten Darstellungen bei Leporin 1725 und Brucker/Haid 1741-1755 (3. Zehend 1744). Eine Fülle autobiographischer Details für den Zeitraum bis 1721 findet sich ferner bei Heister 1753.

5 Zur Person Trews s. Steinke 1999; Schnalke 1997 und Pirson 1953.

6 Trew erwarb grosse Teile der Heister-Korrespondenz aus dem Nachlass seines Lehrers. Damit gelangte er teilweise auch wieder in den Besitz seiner Originalbriefe, vgl. SchmidtHerrling 1940, XXXIVf., und Pirson 1953. - Eine chronologische Übersicht über den Briefwechsel zwischen Heister und Trew findet sich im Anhang zu diesem Beitrag. 


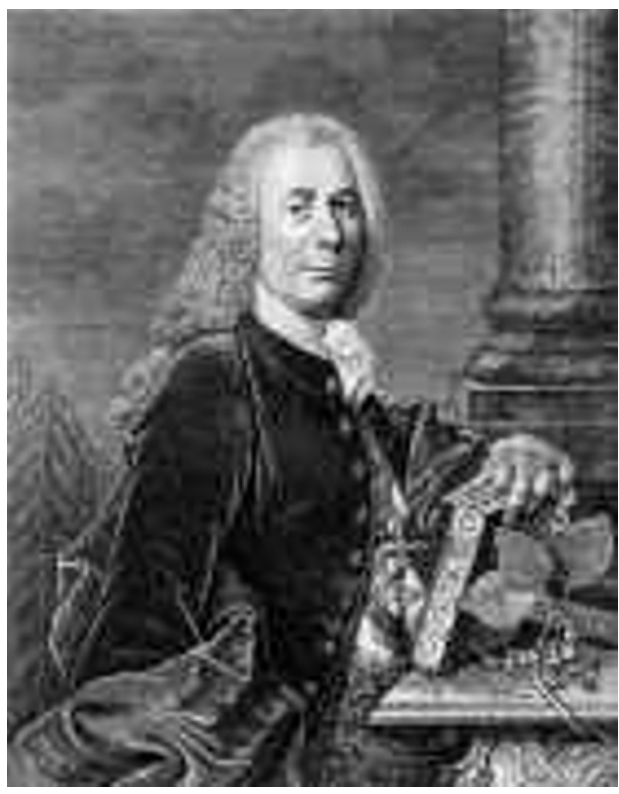

Abb. 2. Christoph Jacob Trew, Kupferstich von Georg Christoph Lichtensteger 1770, nach D. van der Smissen 1748 (Wellcome Library London).
40 Jahre währende Briefwechsel zeigt zeitliche Dichten und brieffreie Intervalle, die es nahelegen, die Entwicklung der Korrespondenz in Phasen zu untersuchen.

\section{Heister und Trew in Altdorf}

Heister, der seit 1710 die Professur für Anatomie, Chirurgie, theoretische und praktische Medizin in Altdorf bekleidet, hat sich in der Fachwelt zunächst vor allem durch seine ophthalmologischen Arbeiten $^{7}$ einen Namen gemacht und legt 1717 sein Compendium anatomicum ${ }^{8}$ sowie 1719 sein umfassendes chirurgisches Lehrbuch ${ }^{9}$ vor - zwei Werke, die seinen wissenschaftlichen Ruhm begründen und entscheidend dazu beitragen, dass die Chirurgie in Deutschland auf der Grundlage einer weit entwickelten Anatomie wieder in den Bereich der akademischen Medizin eingebunden wird.

Christoph Jacob Trew, Sohn des Stadtapothekers von Lauf, einem Städtchen auf dem reichsstädtischen Territorium von Nürnberg, besucht bereits als 15jähriger die Lehrveranstaltungen Heisters, der eben sein Amt an der Nürnberger Universität angetreten hat ${ }^{10}$. Ein Jahr später, im Herbst 1711, immatrikuliert sich Trew offiziell für das Studium der Medizin in Altdorf. Sein Name hat dort einen guten Klang, denn sein Grossvater Abdias Trew (1597-1669), der über dreissig Jahre lang als Professor der Mathematik und Physik an der Nürnberger Universität wirkte, ist als «besondere Zierde dieser Akademie und der ganzen gelehrten Welt» ${ }^{11}$ noch unvergessen. Trew versteht es, sein physikalisches und mathematisches Vorwissen in das Studium einzu-

7 Zu Heisters Bedeutung für die Ophthalmologie s. Ruisinger 2003 und Schlipp 1910.

8 Heister $1717 b$.

9 Heister 1719a.

10 Dass Trew bereits 1710 zu den Schülern Heisters zählte, geht aus dessen Formulierung in dem Gutachten vom November 1712 hervor, in dem er auf Trew als «Auditorem meum iam ultra triennium diligentissimum» Bezug nahm (Universitätsbibliothek Erlangen-Nürnberg, Briefsammlung Trew, Trew Beilage a).

11 Nachwort Heisters zu Trew 1715 (Übersetzung durch Renate Wittern in: Schnalke 1995,185). 
bringen, und macht durch seine raschen Fortschritte in den medizinischen Fächern und seine Begabung im Disputieren auf sich aufmerksam.

Heister gelingt es, das Interesse des begabten und hochmotivierten Studenten auf seine eigenen wissenschaftlichen Forschungsgebiete zu lenken und ihm das methodische Rüstzeug zu vermitteln, das Trew in Zukunft zum eigenständigen Sezieren, Botanisieren und naturhistorischen Sammeln befähigen soll. Zwei erhaltene Dokumente von Trews Hand - die ersten Seiten einer Mitschrift zu Heisters Vorlesung über Verheyens Anatomie des menschlichen Körpers ${ }^{12}$ und ein illustriertes anatomisches Manuskript zur Circulatio sanguinis in foetu ${ }^{13}$, das vermutlich auf eine Sektion Trews unter Heisters Aufsicht zurückgeht - bezeugen nicht nur den guten anatomischen Blick und das zeichnerische Talent Trews, sondern auch die Intensität seiner Einbindung in die Lehre und Forschung Heisters. Wie eng die Beziehung zwischen dem Lehrer und seinem Schüler ist, belegt zudem ein Geschenk, das Trew bereits 1712 von Heister empfängt. Die kleinformatige Kalkplatte mit der Versteinerung eines Knochenfisches soll den jungen Studiosus für den Aufbau einer naturkundlichen Sammlung begeistern, die - über die Grenzen der Anatomie und Botanik hinausgehend - alle drei Reiche der Natur einschliesst ${ }^{14}$.

Im Dezember 1715 verteidigt Christoph Jacob Trew unter dem Vorsitz Heisters seine Inauguraldissertation De chylosi foetus, additis observationibus anatomicis, eine anatomische Studie über das Verdauungssystem beim ungeborenen Menschen, für die er unter Anleitung seines anatomischen Lehrmeisters eigenständige Sektionen durchgeführt hat. Auf der letzten Seite liess der Doktorvater eine äusserst wohlwollende Laudatio auf seinen Schüler einrücken ${ }^{15}$.

Darin bringt Heister jene Charakterzüge des jungen Adepten der Medizin auf den Punkt, die es für ihn interessant erscheinen lassen, mit Trew weiterhin in Kontakt zu bleiben: So spricht er die Talente an, die dieser aufzuweisen hat, und betont insbesondere seine ausgezeichneten «Geistesgaben» und seine «nie erlahmende [...] Gewissenhaftigkeit». Überdies habe der Student eine «große Liebe zu allen Teilen der Medizin» entwickelt. Trew habe sich jedoch nicht verzettelt, sondern im Konzert der Fächer die Anatomie und die Botanik als Interessenschwerpunkte für sich erschlossen und wissenschaftlich kultiviert. Mit höchster Intensität habe er sich in die Themen

12 Vgl. Schnalke 1995, $178 \mathrm{f}$.

13 Vgl. Schnalke 1995, $182 \mathrm{f}$.

14 Vgl. Schnalke 1995, 180.

15 Trew 1715. - Für eine deutsche Übersetzung des von Heister verfassten Nachworts s. Renate Wittern in: Schnalke 1995, 185. 
eingearbeitet und dafür die «Tage und die Nächte» geopfert. Schliesslich attestiert der Professor seinem Studenten all das, was in seinen Augen einen veritablen Forscher ausmacht: Trew nimmt die vorhandene Literatur zur Kenntnis, saugt die Unterweisungen seiner Lehrer in sich auf und prüft das Wahrgenommene eigenständig, ja mit dem Sezier- und Botanisiermesser sogar eigenhändig. Dadurch erarbeitet sich Trew in Heisters Augen den Stand des Wissens auf den Feldern seiner «Lieblingsbeschäftigungen» in umfassender Weise und fügt dem zu errichtenden Gebäude der medizinischen Erkenntnis manchen neuen Baustein hinzu.

Doch an die wissenschaftliche Karriere, die sich Heister von seinem Schüler erwartet, ist für den jungen Doktor der Medizin zunächst nicht zu denken. Anfang 1716 muss Trew Altdorf Hals über Kopf verlassen, nach Lauf zurückkehren und eine ärztliche Praxis eröffnen, um die väterliche Apotheke vor dem Ruin zu retten ${ }^{16}$.

Im Mai 1717 kann sich der Altdorfer Absolvent wieder freimachen. Trew bricht zu einer dreijährigen Studienreise auf, die ihn unter anderem nach Paris, Leiden und Danzig führt, wo er seine anatomischen und botanischen Kenntnisse vertieft und vielfältige Kontakte zu Vertretern der gelehrten Welt knüpft. Dies ist der Zeitpunkt, an dem die uns erhaltene Korrespondenz zwischen Heister und Trew einsetzt.

\section{In Sachen Heister unterwegs (1717-1720)}

Aus der ersten Korrespondenzphase von 1717 bis 1720 haben sich lediglich vier Schreiben Heisters erhalten, in denen dieser das einstige Lehrer-SchülerVerhältnis bekräftigt, um von den Möglichkeiten zu profitieren, die sich Trew in Paris bieten. Denn Heister, den seine eigene Peregrinatio academica zwar in die Niederlande und nach England, nicht aber nach Frankreich geführt hat ${ }^{17}$, verfügt über keine persönlichen Korrespondenten in der Seine-Stadt. Durch genaue Instruktionen, die er Trew mit auf den Weg gibt und in seinen Briefen weiter spezifiziert, versucht der Daheimgebliebene seinen Vertreter vor Ort gezielt zu lenken und zu dirigieren.

Zunächst sieht Heister in Trew den Zulieferer wissenschaftlicher Arbeitsgrundlagen. Bereits vor Reiseantritt beauftragt er ihn, in Paris Fachliteratur zu erwerben, und gibt ihm hierfür «2 Louis d'ors $»^{18}$ mit. In den zwei Schrei-

16 Vgl. Schnalke 1995, 186-199.

17 Eine ausführliche Schilderung des Auslandsaufenthaltes Heisters findet sich bei Leporin $1725,9-17$.

18 Heister 46, 10.12 .1717$. 
ben, die er an Trew in Paris richtet, konkretisiert er seine Bücherwünsche, legt den Briefen Listen mit insgesamt 70 vornehmlich anatomischen und chirurgischen Titeln bei und weist Trew für den Erwerb dieser Werke 120 Gulden an. Sobald er von Trew erfährt, dass dieser auf seiner «Reiß und in Paris $»^{19}$ zahlreiche getrocknete Pflanzen und Samen gesammelt hat, bittet er ihn, ihm davon für sein Herbarium vivum beziehungsweise für den botanischen Garten in Altdorf doppelte oder seltene Stücke zuzusenden. Schliesslich soll Trew durch gezielte Ankäufe zur Erweiterung der umfangreichen Instrumentensammlung seines akademischen Lehrmeisters beitragen.

Heister betreibt zu dieser Zeit intensive Forschungen zur pathologischen Anatomie des Grauen Stars. Es ist ihm daher ein grosses Anliegen, dass Trew für ihn Kontakt zu den Pariser Augenspezialisten aufnimmt, ihnen seine «opuscula $»^{20}$ überreicht und nachforscht, ob sie inzwischen «von frischen und mir noch unbekanden experimentis was gemeldet» haben. Der Altdorfer ist ein überzeugter Verfechter der von Michel Brisseau (1676-1743) und Antoine Maître-Jan (1650-1730) wenige Jahre zuvor formulierten These, dass die Katarakt kein getrübtes Häutchen sei, das vor der Linse schwimme, sondern eine Trübung in der Linse selbst ${ }^{21}$. Sein streitbarer Kontrahent in der Auseinandersetzung um die Starfrage, der englische Okulist John Thomas Woolhouse (um 1650-1734), lebt ebenfalls in Paris. Auch über ihn zieht Heister Erkundigungen ein und bittet Trew, ihm mitzuteilen, ob er mit diesem «nicht bekand [ist], und was [er] bey Ihm gesehen» hat.

In diesem Zusammenhang steht ein weiterer Auftrag Heisters an Trew: Dieser soll anhand des reichen Leichenmaterials, das ihm in Paris zur Verfügung steht, eine Frage prüfen, von deren Klärung er ein gewichtiges Argument zugunsten der neuen Starlehre erhofft ${ }^{22}$. Er bittet Trew, in seinem Auftrag Gefrierschnitte von Menschenaugen anzufertigen und sorgfältig zu vermessen. Er selbst habe in Altdorf dieses Experiment nur an den Augen von Schweinen und Hunden durchführen können, weil er «bey der stärksten Kälte damahls eben keine von Menschen habe bekommen können $»^{23}$.

Auch zu anderen Fragestellungen sendet Heister anatomische Arbeitsaufträge nach Paris. So fordert er Trew auf nachzuforschen, «in welchem musculo Sie die ossicula sesamoidea in femore finden», und regt ihn an, der

19 Dieses und die folgenden Zitate aus Heister 47, Anfang 1718. - Eine auszugsweise Veröffentlichung dieses Schreibens findet sich als Brief Heisters «nach Paris mit unbek. Adr.» bei Schlipp 1910, 410f.

20 Gemeint sind wohl die beiden Katarakt-Traktate: Heister 1713,1717a.

21 Zur Positionierung Heisters im Streit um den Star vgl. ausführlich Ruisinger 2003.

$22 \mathrm{Vgl}$. Heister 1753, 742-745.

23 Heister 1753, 743. 
Behauptung des Turiner Anatomen Giovanni Battista Bianchi (1681-1761) nachzugehen, dass es keine «valvulam coli» gebe und dass das Ileum «nicht in sinistrum parietem coli sondern in dextrum, und zwar ad angulum rectum» inseriere. Er selbst zweifelt daran, hat aber «kein cadaver gehabt, umb wegen dießer Sach selbst nachzusehen». Zuletzt bittet er Trew noch um die Anfertigung und Zusendung eines anatomischen Präparats:

Schlüßlich, weil mir von einem sceleto die pedes extremi verdorben, so wolte gebeten haben, umb solches zu completiren, von einem starcken Kerl ein paar solche Füße nehmlich nur tarsum, metatarsum et digitos pedis utriusque so zu praepariren, daß ich selbige zum dem sceleto gebrauchen könte, und entweder zu den büchern und instrumenten zu packen, oder auf andere manier zu übersenden.

Trew soll in Paris nicht nur durch gezielte Sektionen neue Erkenntnisse für Heister gewinnen, sondern auch dort kursierende wissenschaftliche Neuigkeiten nach Altdorf weiterleiten ${ }^{24}$. Insbesondere fragt Heister nach, «was etwa neues in Re medica zu paris passiret $»^{25}$ und ob sein Schüler «keine experimenta de cataracta gesehen [habe], als worauf fleißig achtzugeben bitte» ${ }^{26}$. Im Gegenzug teilt er selbst eine ophthalmologische Beobachtung aus seiner forensischen Tätigkeit mit: In Altdorf habe sich zugetragen, «daß vorigen october H. Schut Studiosus nachts erstochen worden, und weilen derselben einen cataractum verum am lincken Aug gehabt, so habe bey visitatione vulneris auch das Aug geöffnet, und nichts anders als crystallinum opacum gefunden $»^{27}$.

Trew wird den vielfältigen Erwartungen, die sein akademischer Lehrer in ihn setzt, nur zum Teil gerecht. Die von Heister erhoffte regelmässige, in einem 10- bis 12tägigen Rhythmus eingerichtete Korrespondenz baut sein einstiger Schüler nicht auf ${ }^{28}$. Vermutlich blieb es bei den zwei Briefen, auf die sich Heister bezieht ${ }^{29}$. Darin informiert Trew den Altdorfer Professor offensichtlich über seine botanischen Sammlungserfolge und zeigt ihm den Ankauf und die Zusendung der gewünschten Bücher an. Vermutlich hat Trew die Rolle eines wissenschaftlichen Agenten für Heister in Paris nie in einem weitergehenden Umfang ausgeübt, da weder er noch Heister in ihren späte-

24 Vgl. Heister 45, 9.2.1717.

25 Heister 46, 10.12.1717.

26 Heister 47, Anfang 1718.

27 Heister 46, 10.12.1717. - Diese Kasuistik erscheint als 500. Wahrnehmung in der von Heister herausgegebenen Fallsammlung, s. Heister 1753, 847-850.

28 Heister hielt Trew das Beispiel von Johann Wilhelm Widmann I (1690-1743) und anderen Ärzten aus Nürnberg vor Augen, die in der gleichen Situation ihren Korrespondentenpflichten wesentlich häufiger nachgekommen seien. - Von Widmann I sind Briefe an Heister aus Strassburg und Paris erhalten (Widmann I 1, 10.1.1713;2, 1.6.1713).

29 Heister erwähnte einen Brief Trews vom 30.12.1717 (Heister 47, Anfang 1718) sowie ein Schreiben seines Schülers vom 10.2.1720 (Heister 48, 5.3.1720). - Keines der beiden Schriftstücke lässt sich in der Erlanger Universitätsbibliothek nachweisen. 
ren Briefen auf diese Angelegenheiten zurückkommen. Auch die ophthalmologischen Auftragssektionen dürfte Trew niemals durchgeführt haben ${ }^{30}$.

Dennoch empfiehlt Heister den jungen Arzt im Jahre 1720 als seinen Nachfolger in Altdorf. Darin mag sich die starke persönliche Bindung und wissenschaftliche Wertschätzung des Lehrers gegenüber seinem Schüler ausdrücken. Allerdings kann Heister in diesem Zusammenhang die Enttäuschung über dessen nachlässige Korrespondenzführung nicht verleugnen:

[...] und ob Sie schon offt sehr saumseelig in Ihrem schreiben an mich gewesen, so bin ich dennoch nicht saumseelig gewesen, um Sie an meine Stelle best möglich zu recommendiren. Ich habe aber meister orthen bemercket, daß sie durch dieße Negligence im schreiben, so wohl an Ihre Patrone, als Mutter und andere Freunde, sich bey vielen übel recommendirt haben, und wenn nichts aus der Sache wird, so können Sie es dießem Fehler zuschreiben. ${ }^{31}$

Heisters Vorahnung bestätigt sich. Trew unterliegt in der Bewerbung um die Altdorfer Professur seinem Konkurrenten Johann Heinrich Schulze (1687-1744) aus Halle und lässt sich statt dessen in Nürnberg nieder. Mit dieser Absage an eine akademische Laufbahn verlässt er die eigentliche Sphäre Heisters. Es gelingt ihm aber, auch ausserhalb des universitären Lebens seine wissenschaftlichen Schwerpunkte, die Anatomie und die Botanik, weiter auszubauen. Das bisherige Patronageverhältnis zwischen Heister und Trew verliert in der Folge an Bedeutung und wird durch ein neues, auf gemeinsamen wissenschaftlichen Interessen und Bedürfnissen basierendes Briefverhältnis abgelöst. Dabei erkennt Trew die fachliche Autorität seines ehemaligen Lehrers uneingeschränkt an, der sich zwischenzeitlich in Helmstedt als angesehener Professor und hochgeschätzter Arzt und Operateur etabliert hat. Trew legt seinen Lehrveranstaltungen, die er auf dem Nürnberger Theatrum anatomicum abhält, die einschlägigen Schriften Heisters zugrunde $^{32}$ und tritt wie dieser für ein iatromechanistisches Körperbild und eine «subtile Anatomie» ein ${ }^{33}$.

30 Vgl. Heister 1753, 744, wo derartige Gefrierschnitt-Untersuchungen erwähnt werden, aber nicht eine Beteiligung Trews.

31 Heister 48, 5.3.1720.

32 So begründete Trew einen chirurgischen Kurs damit, dass «einigen von denen Herren Chirurgis beliebet, eine Explication deß ersten theils der Chirurgie Herrn Dr. Heisters, welcher von Wunden, Geschwulsten, geschwühren, Beinbrüchen und Veränckungen handelt, von mir zu hören [...]» (Universitätsbibliothek Erlangen-Nürnberg, Briefsammlung Trew, Trew Beilage b, 17.3.1723). - Vgl. Schnalke 1995, 54f., 218-221.

33 Vgl. Trew 1724, Trew 1729. - Für Heister vgl. Boschung 1983, $35 f$. 


\section{Die Emanzipation des Schülers (1730-1736)}

Die erhaltene Korrespondenz setzt erst wieder mit einem Schreiben Heisters vom 12. Mai 1730 ein. Offenbar stehen Heister und Trew zu diesem Zeitpunkt bereits wieder seit längerem in Kontakt, da sich Heister in seinem Brief relativ beiläufig für eine Zusendung von Pflanzensamen bedankt. Dass die Korrespondenz 1730 deutlich belebt und in einer grösseren Briefdichte bis 1736 fortgeführt wird ${ }^{34}$, liegt in einer gleichsinnigen Verlagerung der wissenschaftlichen Arbeitsschwerpunkte der Briefpartner begründet, die das bisherige Autoritätsverhältnis zwischen Trew und Heister aufbricht. Während Trew sein anatomisches Lehrengagement reduziert und seine botanische Studien- und Sammlungstätigkeit intensiviert, legt Heister seine anatomische Professur nieder und übernimmt neben der theoretischen Medizin auch den Lehrauftrag für Botanik. Die Botanik wird nun zu dem Gebiet, auf dem Trew und Heister sich treffen.

Der Jüngere lässt sich zunächst durch seinen früheren Lehrer erneut in die Pflicht nehmen. Heister ist daran gelegen, den vernachlässigten Hortus medicus von Helmstedt wieder instand zu setzen und auszubauen ${ }^{35}$. Er bittet Trew um Pflanzensamen, Knollen, Gewächse und Kräuter ${ }^{36}$ sowie um einschlägige Literatur. Der Nürnberger vermittelt auch Kontakte zu bedeutenden Botanikern in Bern (Albrecht von Haller), Paris (Bernard und Antoine de Jussieu) und Pisa (Michelangelo Tilli), lässt Heister aber so lange auf die Sendungen warten, dass dieser erneut die saumselige Korrespondenzführung Trews beklagen muss ${ }^{37}$. Das Schwergewicht beim Austausch botanischer Produkte bleibt dabei stets auf Heisters Seite. Er ist der Fordernde und Nehmende ${ }^{38}$.

34 Gegenüber den 21 Briefen Heisters haben sich zwar lediglich 7 Briefe oder Briefentwürfe Trews erhalten, jedoch nahm Heister in seinen Schreiben auf weitere 4 Zusendungen und 6 Briefe Trews Bezug.

35 Heister klagte in seinem Brief vom 12.5.1730: «Es siehet bißdato in unserem horto medico gar schlecht aus, weil mein antecessor denselben nicht geachtet, sondern gantz negligiret.»

36 Mit Ausnahme des kurzen Schreibens vom 15.5.1733 (Heister 63) enthalten alle Briefe Heisters aus dieser Korrespondenzphase Anmerkungen zur Beschaffung von botanischen Produkten.

37 Heister 59, 3.12.1731; Heister 64, 9.11.1733. Besonders gegen Ende dieser Korrespondenzphase nahmen Heisters Klagen zu: «Ich habe jetzo fast in 2 Jahren nicht mehr die Ehre gehabt eine antwort auf verschiedenere meiner schreiben zu erhalten, und vermuthe daraus, daß ich gantz bey Ihnen in das vergeßen gerathen [...]» (Heister 65, 19.4.1734), sowie: «Ob ich schon lang keine briefe noch sonsten was von Ihnen erhalten, und es scheinet, als ob ich gantz bey Ihnen in das vergeßen komme [...]», Heister 69, 30.1.1736.

38 Heister verwandelte nicht zuletzt dank der Unterstützung durch sein dicht gewebtes Korrespondentennetz den medizinischen Garten in Helmstedt in wenigen Jahren zu einem reich bestückten botanischen Studienort, dem Trew bald kaum noch etwas hinzuzufügen wusste: «Der Vorrath so Euer HochEdelgebohren allbereit besiezen ist so schon so groß, daß es schwehr fällt dem selben etwas bey zu sezen, zumahlen einem so kleinen winckel Gärtner wie ich binn.» Trew 326, 26.4.1732. 
Dieses Ungleichgewicht erfährt auf zwei Ebenen einen Ausgleich: Zum einen weiss Heister um den bibliophilen Sammeleifer Trews und bietet sich mehrfach dazu an, für ihn Bücher zu ersteigern ${ }^{39}$. Zum anderen, und dies dürfte zu diesem Zeitpunkt für Trew wichtiger gewesen sein, eröffnet sich für den Nürnberger Arzt durch seine Dienstfertigkeit die Möglichkeit, mit Heister einen gleichrangigen fachwissenschaftlichen Austausch zu führen. So teilt Heister Trew mit, welche Samen in seinem Garten aufgegangen sind ${ }^{40}$ und welche nicht ${ }^{41}$ und was für Beobachtungen er beim Studium seiner Pflanzen gemacht hat ${ }^{42}$. Er sendet seine Bestimmung einiger neuer Pflanzen nach Nürnberg und bittet Trew um sein fachliches Urteil, «ob ich ursach gehabt neue genera aus bemeldeten pflantzen zu machen oder nicht ${ }^{43}$. Daraufhin entspinnt sich ein gelehrter Dialog, in dem Trew sich durch seine eigenen Beobachtungen und seine profunde Kenntnis der Fachliteratur als ernstzunehmender Botaniker präsentiert ${ }^{44}$.

Trew meldet auch eigene Beiträge zur Systematisierung der Pflanzenwelt nach Helmstedt: «Ich habe in dem achten Specimine auch 2 neue Genera plantarum proponiret ${ }^{45}$, wie solche werden aufgenommen werden muß ich erwarten. ${ }^{\star 6}$ Heister liest die botanischen Publikationen seines ehemaligen Schülers sehr aufmerksam durch und knüpft in seinen Briefen bisweilen direkt daran an. So bezieht er sich etwa auf Trews Beschreibung der Blüte eines Säulenkaktus (Flos cerei) und bittet um Erläuterung, ob die Pflanze im Freiland oder im Gewächshaus gestanden habe ${ }^{47}$.

Das Interesse Trews an einem botanischen Dialog mit Heister kommt ferner darin zum Ausdruck, dass er diesen nicht nur auf seine bereits veröffentlichten Arbeiten aufmerksam macht, sondern ihm auch Rohfassungen seiner botanischen Aufsätze zur kritischen Würdigung vorlegt. So sendet er eine «abzeichnung von der orchioide, und eine eintheilung Derer orchidum wie ich solche gemacht» nach Helmstedt und bittet Heister: «Wollen Sie Dero Gutachten mir darüber ertheilen werden Sie mich gar sehr obligiren.» ${ }^{48}$ Dieser antwortet darauf, dass ihm «Genus und Abzeichnung ihrer orchioidis» gefalle und er es als notwendig ansehe, dass noch weitere neue «generibus

39 Heister 49, 12.5.1730; Trew 321, 1.7.1730; Heister 51, 4.8.1730; Heister 52, 12.9.1730; Heister 53,17.10.1730; Heister 54, 1.12.1730; Trew 322, o.D. (1731?).

40 Heister 66, 12.7.1734.

41 Heister 52, 12.9.1730; Heister 64, 9.11.1733.

42 Heister 51, 4.8.1730.

43 Heister 54, 1.12.1730.

44 Trew 323, 23.2.1731; Heister 56, 28.3.1731.

45 Trew 1731.

46 Trew 323, 23.2.1731.

47 Heister 55, 29.12.1730.

48 Trew 323, 23.2.1731. 
müßen gemacht werden, um die botanique in beßere deutlichkeit zu setzen ${ }^{49}$. Schliesslich bemüht sich Trew sogar um eine Revision der botanischen Terminologie:

Ich habe nun einen catalogum davon dergestallt unter handen daß ich nicht nur alleine meine eigene observationes circa characteres genericas beysezen, sondern auch eine Probe geben will, wie nüzlich es wäre wann in enumeratione plantarum einer jeden ihr character specificus kürzlich bey gesellet würde. ${ }^{50}$

Trew nimmt damit zu diesem Zeitpunkt der Korrespondenz gegenüber Heister die Position eines wissenschaftlich originär denkenden und produzierenden Forschers ein, der an der allgemeinen Entwicklung der Botanik hin zu einer systematisierenden, sich morphologisch ausrichtenden Naturwissenschaft mitwirkt ${ }^{51}$.

Mit der Gründung des Commercium litterarium, der ersten medizinischen Wochenschrift, gewinnt Trew gegenüber Heister noch mehr an Gewicht ${ }^{52}$. Er ist für Heister nun nicht nur botanischer Zulieferer und fachlicher Gesprächspartner, sondern auch wissenschaftlicher Redakteur und Mediator. Trew schafft sich und seinem Briefpartner mit dieser Zeitschrift ein Forum, auf dem sie die Ergebnisse ihres wissenschaftlichen Austauschs veröffentlichen können. Heister ist daran gelegen, das Periodikum nach Kräften zu fördern und zu nützen. Er erklärt sich bereit, «gern öffters was [zu] contribuiren ${ }^{53}$, und bietet seine Kontakte zu angesehenen Fachkollegen an, um der Zeitschrift eine gute Aufnahme und grössere Verbreitung zu sichern ${ }^{54}$. Er kommentiert das Erscheinen des Commercium litterarium mit kritischen Anmerkungen, Richtigstellungen und Verbesserungsvorschlägen ${ }^{55}$. Trew erhält von ihm nicht nur seine gedruckten Pflanzenkataloge, Disputationes und

49 Heister 56, 28.3.1731.

50 Trew 327, 5.10.1732.

51 Trews Leistungen auf botanischem Gebiet wurden bislang fast ausschliesslich in der Herausgabe illustrierter Pflanzenwerke gesehen; vgl. Wegner 1940; Nissen 1951; Mann 1964. Eine umfassende Würdigung seiner Bedeutung als wissenschaftlicher Botaniker steht noch aus; erste Ansätze liefern Rees 1884; Spiess 1890.

52 Der vollständige Titel des Organs lautet Commercium litterarium ad rei medicae et scientiae naturalis incrementum. Zusammen mit vier Kollegen aus der Nürnberger Ärzteschaft und dem Altdorfer Medizinprofessor Johann Heinrich Schulze gründete Trew 1730 eine Sozietät, welche ab 1731 die Herausgabe der Zeitschrift besorgte. Trotz wachsender verlegerischer und vertrieblicher Schwierigkeiten konnte sich das Journal - zeitweise in der europäischen Gelehrtenrepublik hochgeachtet - bis 1745 halten; vgl. dazu Pirson 1953, 477-490. Derzeit bereitet Tilmann Rau in einem Erlanger Dissertationsprojekt eine erste fundierte Studie zum Commercium litterarium vor.

53 Heister 55, 29.12.1730.

54 Heister 55, 29.12.1730.

55 So merkte Heister mit Hinblick auf die Acta, das Journal der Leopoldina, an: «[...] und vermeine ich gleichfals, daß daßelbige [Commercium litterarium] so einzurichten, daß es die Acta Societatis Naturae Curiosorum nicht über den haufen werfen möge: welches etwa onmaßgeblich wird geschehen können, wenn in dem Novo instituto man dergleichen observationes, welche man sonsten in bemeldete acta gesetzt, nicht gantz und vollkommen, 
Programmata zur Rezension in diesem Journal zugesand $t^{56}$, sondern Heister vermittelt auch die Schriften seines Sohnes Elias Friedrich (1715-1740) ${ }^{57}$ und des in Petersburg tätigen Anatomen, Physiologen und Botanikers Josias Weitbrecht (1702-1747) an seinen Nürnberger Korrespondenten ${ }^{58}$.

Der wissenschaftliche Austausch zwischen Trew und Heister konzentriert sich zu diesem Zeitpunkt des Briefwechsels zwar auf botanische Themen, die ganze Breite des fachlichen Dialogs zeigt sich jedoch in den beruflichen sowie im engeren Sinne medizinischen Mitteilungen, die eher beiläufig in die Briefe eingestreut sind. So informiert Trew seinen Briefpartner über einige Krankheitsverläufe bei Patienten, die er Heister 1730 persönlich vorgestellt hatte, als dieser zu einem Besuch in Nürnberg weilte. Heister gibt Trew dagegen Einblicke in sein Wirken an der Universität Helmstedt. Er stöhnt unter der Arbeitsbelastung, die eine erneute Übernahme des Rektorats 1733 mit sich bringt ${ }^{59}$, und äussert sich wiederholt über sein gespanntes Verhältnis zu Peter Gericke (1693-1750), der seit 1730 den Lehrstuhl für Anatomie in Helmstedt innehat ${ }^{60}$. Er unterrichtet Trew mehrmals über die Neuauflagen seiner anatomischen und chirurgischen Werke ${ }^{61}$, teilt dem Nürnberger mit, wann er wieder vermehrt seziert und daher mit anatomischen Observationen aufwarten $\mathrm{kann}^{62}$, wie sich die Zusammenarbeit mit seinem Sohn entwickelt ${ }^{63}$ und welchen Werdegang dieser einschlägt ${ }^{64}$. Dabei kommt Heister zu allgemeinen Aussagen über die medizinischen Ausbildungsverhältnisse in Deutschland: «Mein Sohn ist schon seit Michaelis in Berlin, um für anatomia und chirurgica alda zu tractiren, und sich darinnen zu perfectioniren, indem man jetzo allda eben so gute Gelegenheit fast hat als zu Paris oder anderwerts, auch allzeit cadavera genug alda sind, in gleichen viele patienten und operationes in der charité.» ${ }^{65}$

sondern nur die contenta oder Hauptsache brevissimis referirt, und meldet daß die observation vollkommen in den Actis solle vorkommen, so thäte eines dem andern keinen schaden. Recensiones von Büchern und dissertationibus haben Sie besonders, welche ohne dem nicht in die Acta kommen [...].» Heister 56, 28.3.1731. - Mit dieser Bitte entsprach Heister einer Aufforderung des Leopoldina-Präsidenten Johann Jacob Baier, die Nürnberger Ärzte mögen mit dem Commercium litterarium keine Konkurrenz zum Leopoldina-Organ etablieren. Als sich Trew und seine Herausgeberkollegen weigerten, Baiers Ansuchen zu entsprechen, rief Baier zum offenen Boykott der Nürnberger Zeitschrift auf. Vgl. in diesem Zusammenhang Schnalke 2002,100f.

56 Trew 323, 23.2.1731; Heister 56, 28.3.1731; Trew 327, 5.10.1732.

57 Heister 63, 15.5.1733.

58 Heister 66, 12.7.1734.

59 Heister 61, 5.1.1733.

60 Zum Konflikt zwischen Heister und Gericke siehe auch Stukenbrock 2001,127,153,169f., 183.

61 Heister 50, 30.6.1730; Heister 57, 11.5.1731; Heister 58, 21.8.1731; Heister 59, 3.12.1731.

62 Heister 49, 12.5.1730.

63 Heister 68, 4.3.1735.

64 Heister 69, 30.1.1736.

65 Heister 69, 30.1.1736. 
Als an der kurz vor ihrer Inauguration stehenden Universität Göttingen die medizinischen Lehrstühle besetzt werden sollen, zeigt sich, wie hoch und ungebrochen die fachliche Wertschätzung Heisters für Trew ist: Nachdem er sich zunächst selbst mit dem Gedanken getragen hatte, seinen Wirkungskreis nach Göttingen zu verlegen, empfiehlt er statt dessen 1734 seinen Schüler für das akademische $\mathrm{Amt}^{66}$. Als auch dieser kein Interesse signalisiert, fällt in Hannover die Wahl schlussendlich auf einen jungen, aber vielversprechenden Schweizer Arzt: Albrecht von Haller (1708-1777).

Der Briefwechsel eröffnet in dieser Phase den Blick auf eine Dimension des Verhältnisses zwischen Trew und Heister, die im weiteren Verlauf der Korrespondenz noch an Bedeutung gewinnen wird: die private Sphäre. So zeigt Heister dem Nürnberger nicht nur den Tod seiner Mutter und seines Schwagers $a^{67}$, sondern wartet auch mit der Nachricht von der Hochzeit seiner ältesten Tochter auf ${ }^{68}$.

Auf diese Phase eines verhältnismässig regen Briefwechsels folgen knapp sechs Jahre, aus denen sich keine Briefe erhalten haben. Der Kontakt zwischen Trew und Heister dürfte in dieser Zeit jedoch nicht abgerissen sein. Der vertraute Ton, der die Briefe ab 1742 weiterhin kennzeichnet, lässt keinen Bruch vermuten, und die Briefthemen knüpfen nahtlos an die bisherige inhaltliche Entwicklung der Korrespondenz an.

\section{Botanischer Dialog auf Augenhöhe (1742-1746)}

Die dritte Phase des Briefwechsels umfasst die Jahre 1742 bis 1746. Trew hat in der Zwischenzeit die Osteologie des Schädels als Fragment des ersten Teils seines auf mehrere Bände projektierten anatomischen Tafelwerks vorgelegt ${ }^{69}$. Er führt neben seiner gutgehenden Praxis unter grossen Mühen das Commercium litterarium bis 1745 weiter und bemüht sich um die Vergrösserung seiner Bibliothek und seiner Sammlung botanischer Illustrationen.

Heister tauscht 1740 seinen Lehrauftrag für theoretische mit jenem für praktische Medizin. Daneben lehrt er weiterhin die Chirurgie und vor allem die Botanik, wobei sein besonderes Engagement nach wie vor dem Ausbau des akademischen Gartens von Helmstedt gilt. Er äussert sich in einer Reihe von Schriften zu den theoretischen Grundlagen der Medizin und weist dabei

66 Heister 65, 19.4.1734; Heister 66, 12.7.1734; Heister 67, 30.8.1734. - Vgl. in diesem Zusammenhang auch Schnalke 1994.

67 Heister 56, 28.3.1731.

68 Heister 68, 4.3.1735.

69 Trew 1740. 
insbesondere einer auf soliden anatomischen Kenntnissen fussenden Wundarznei einen höheren Rang innerhalb der Medizin $\mathbf{z u}^{70}$. Die Verbindung seines Wirkens als praktisch tätiger Arzt, anatomischer Forscher, medizinischer Theoretiker und akademischer Lehrer, in der sich Erfahrung, heilkundige Fertigkeit, Belesenheit und experimentelle Neigung auf dem Boden seines iatromechanistischen Weltbildes ergänzen, begründet seinen Ruf als pragmatische Autorität in der deutschen Medizin um die Mitte des 18. Jahrhunderts ${ }^{71}$.

Der Briefwechsel dieser Jahre ist gekennzeichnet durch die Kontinuität im fachlichen Gedankenaustausch sowie durch häufigere Mitteilungen aus der privaten Lebenssphäre. Der Ton der Briefe verrät eine zunehmende Vertrautheit und eine steigende Hochachtung für die Persönlichkeit des Gegenübers. So entschuldigt sich Trew zwar noch für seine unregelmässige Korrespondenzführung: «Meine weitläuffige praxis, oftmaliges Verreisen und die vornehmlich auf mir liegende Besorgung des commercii litterarii, hintert mich an vielem, was ich doch gar wohl für nothwendig erkenne.» ${ }^{72}$ Doch Heister reagiert nun nicht mehr mit Vorwürfen, sondern bekennt sich zu denselben Hinderungsgründen:

Die Ursachen, welche Euer HochEdelgebohren voriges Jahr, warum Sie so lang nicht an mich geschrieben hatten, gemeldet nehmlich ihre viele geschäfften und verhinderungen, werden Sie hoffentlich auch bei mir gelten laßen, da ich mit laboribus academicis, practicis, vielen correspondentzen etc. so sehr überhäufft bin, als wohl jemand. ${ }^{73}$

Botanische Briefgedanken bilden nach wie vor einen thematischen Schwerpunkt der Korrespondenz. Die im engeren Sinne medizinischen Briefinhalte gewinnen aber stärker an Gewicht. Während Heister das erstmalige Erscheinen seines Compendium medicinae practicae ${ }^{74}$ sowie die Neuauflage seines chirurgischen Lehrbuchs ${ }^{75}$ nach Nürnberg meldet, äussert sich Trew über das weitere Schicksal seines anatomischen Tafelwerks. Hofft er zunächst noch, das gesamte Opus zu einem würdigen Abschluss zu bringen ${ }^{76}$, vermerkt er etwas später mit einem Unterton der Skepsis: «[...] was in den übrigen theilen noch werde praestiren können, mus die Zeit geben.» ${ }^{77}$

Heister hält sich mit Anfragen bezüglich der ausbleibenden Fortsetzung der Trewschen Anatomie zurück und klagt auch nicht über die Defekte des

70 Zur Aufwertung der Wundarznei vgl. Heister 1736.

71 Vgl. Rosen 1975, 387.

72 Trew 329, 2.4.1742.

73 Heister 70, 25.3.1743.

74 Heister 70, 25.3.1743; Heister 1744.

75 Heister 73, 31.3.1746.

76 Trew 329, 2.4.1742.

77 Trew 332, 9.5.1744. 
Commercium litterarium. Im Gegenteil, er bringt seine grosse Wertschätzung für die Zeitschrift wiederholt zum Ausdruck und schickt fortwährend die unter ihm gehaltenen und weitgehend von ihm verfassten Disputationen ein, die in der Zeitschrift häufig auch referiert werden. Noch 1746 bemüht er sich, über das Periodikum auf die Vorzüge einer Lithotomietechnik aufmerksam zu machen, die sich bei ihm in der Praxis bewährt habe, die «in Franckreich, England und meisten andern Orten» allerdings verachtet werde ${ }^{78}$. Dabei handelte es sich um den Blasensteinschnitt nach Celsus, ein Verfahren, das nicht zuletzt deswegen in Misskredit geraten war, weil es wenig Ansprüche an das Instrumentarium des Operateurs stellte und sich deshalb bei den herumziehenden Steinschneidern grosser Beliebtheit erfreute ${ }^{79}$. Er ziehe es anderen Vorgehensweisen vor und habe deshalb den Stellenwert der Celsianischen Methode in einer Dissertation von Johann Wilhelm Ilsemann verteidigen lassen ${ }^{80}$. Freilich kommen, so klagt Heister, «teutsche disputationes selten in fremde länder [...]». Er habe daher einen «zimlich weitläufftigen extract von selbiger machen laßen, welchen hierbei übersende, und ersuche ihn in das Commercium litterarium inseriren zu laßen; Damit dadurch dieses scriptum apud exteros bekant werden möge» ${ }^{81}$.

An diesem Beispiel wird deutlich, dass Heister die besondere Bedeutung des Commercium litterarium in seiner internationalen Rezeption sieht. Damit schätzt er Trew als Wissensmultiplikator, der entscheidend dazu beiträgt, dass die Ergebnisse der medizinischen Forschung in Deutschland auch in anderen europäischen Ländern wahrgenommen werden.

Schliesslich bietet Heister seinem Nürnberger Briefpartner auch noch zu Themen der praktischen Medizin jenen gleichberechtigten Dialog an, den er mit ihm auf dem Gebiet der Botanik bereits seit langem führt. Er schickt den ausführlichen Auszug seiner Abhandlung über das Steinschnittverfahren Trew nicht nur zur Publikation, sondern auch zur kritischen Stellungnahme zu. Damit steht Trew im gesamten medizinischen Fachdiskurs mit Heister auf einer Stufe ${ }^{82}$.

Gleichzeitig intensiviert sich der persönlich-private Dialog zwischen den Korrespondenten. Die Brücken zwischen den Welten des Berufs und der Familie schlagen Heisters Mitteilungen von den Ereignissen an der Universität Helmstedt und Trews Empfehlungen für Nürnberger Medizinstudenten.

78 Heister 73, 31.3.1746.

79 Zur Modifikation der Lithotomieverfahren zu Anfang des 18. Jahrhunderts s. Ruisinger 2004b.

80 Heister/Ilsemann 1745.

81 Heister 73, 31.3.1746.

82 Heister 73, 31.3.1746. 
Heister meldet Trew die Berufung Johann Friedrich Crells (1707-1747), seines künftigen Schwiegersohns, nach Helmstedt ${ }^{83}$. Er berichtet die endgültige rechtliche Abtretung der Universität durch den englischen König an den Herzog von Braunschweig und verknüpft damit indirekt die Hoffnung, dass sich seine berufliche Situation noch weiter verbessern werde ${ }^{84}$. Schliesslich schreibt er nach Nürnberg: «Nachdem vor 4 Wochen als den 28 Februarii unser alter Senior Probst von der Hardt im 86 Jahr gestorben, bin ich nunmehr dadurch Senior totus Academiae geworden.» ${ }^{85}$ Trew seinerseits verwendet sich für zwei Nürnberger Medizinstudenten, die in Helmstedt unter Heister ihre Ausbildung abschliessen wollen ${ }^{86}$. In diesem Zusammenhang drückt er seine hohe Wertschätzung für Heister als medizinischen Lehrer aus. So würden sich all jene, die mit «Ernst und Fleiß» unter Heister studieren, «bey Euer HochEdelgebohren [...] selbsten recommendiren ${ }^{87}$.

Ein Student aus dieser Gruppe, Johann Wilhelm Widmann II (1721-1766), findet in den Briefen häufiger Erwähnung. Sein Vater Johann Wilhelm Widmann I (1690-1743), Mitglied des Nürnberger Collegium medicum und hochrangiger Leopoldina-Repräsentant, ist beiden Korrespondenten freundschaftlich verbunden. Der junge Studiosus hat im Hause Heisters Kost und Logis gefunden. Er korrespondiert selbst mit Trew und übermittelt seinem Hausherrn gelegentlich Sendungen und Grüsse des Nürnbergers. Als sein Vater am 16. Juni 1743 stirbt, will er «wegen seiner hier angefangenen Collegiorum nicht nach hauß vor jetzo, und befindet sich, nach dem Er sich von der allzugrosen betrübnus was erhohlt, anjetzo zimlich wohl» ${ }^{88}$.

83 Heister 100, 20.11.1741. - Zu Crell vgl. Triebs 1995, 48f., sowie die zu seinem Gedenken herausgegebenen Leichencarmina, Universität Helmstedt 1747.

84 Heister 72, 8.4.1745.

85 Heister 73, 31.3.1746.

86 Es handelte sich dabei um «des Herrn Bauridels ehemaligen Pfarrers in Rasch, nachmals hiesigen Diaconi bey St. Egydien sein Herr Sohn, der eine Zeitlang in Halle medicinam studiret und nun sonderlich unter Euer HochEdelgeb. direction seine studia zu absolviren wünschet $[\ldots]$ ( Trew 331, 15.9.1742) und um einen nicht näher belegbaren «Herrn Bruckner» (Trew 332, 9.5.1744).

87 Trew 331, 15.9.1742.

88 Heister 71, 28.6.1743. - Wenig später schrieb Widmann II an Trew, es sei ihm wohl bekannt, dass diesen eine enge Freundschaft mit seinem Vater insbesondere in dessen letzter Krankheitszeit verbunden hatte, wofür er ergebenst dankte. Er bat Trew, ihm die gleiche Gütigkeit angedeihen zu lassen, sein Gönner zu bleiben «und mir bey vorkommenden Gelegenheiten deroselben klugen Rath und kräftigen Beystand zu ertheilen». Er werde es seinerseits nicht versäumen, seine «Studia mit solchem Eifer zu treiben [...] Besonders habe mir auch von Herrn Hoffrath Heister und Frau Hoffräthin (als welche sich Ew. HochEdelgebohren ergebenst empfehlen lassen) grosse Hülfe und Beystand zu versprechen, indem mir dieselbe sehr viele Höflichkeit erzeigen, und recht vätterlich vor mich sorgen» Widmann II 2, 15.7.1743. 
Der Tod von Johann Wilhelm Widmann I ist für Heister Anlass, Trew in einer familiären Rechtsangelegenheit um einen Freundschaftsdienst zu bitten. Der Verstorbene war von Heister damit beauftragt worden, im Falle des Todes seiner im Nürnberger Land lebenden Schwiegermutter in dem zu erwartenden Erbschaftsstreit die Interessen der Familie Heister zu vertreten $^{89}$. Trew willigt ein, sich diese Aufgabe übertragen zu lassen, und nimmt die von Widmann hinterlassenen Dokumente in Verwahrung ${ }^{90}$. Bald schon sollten die Korrespondenten auf diese Angelegenheit zurückkommen.

\section{Breiter Gedankenaustausch in beruflichen und privaten Belangen (1747-1751)}

Der Briefwechsel zwischen Trew und Heister tritt zwischen 1747 und 1751 in seine intensivste Phase. Den erhaltenen 21 Schreiben Heisters und zwei Briefen von Trews Hand sind wenigstens noch acht Schriftstücke des Nürnberger Arztes hinzuzurechnen, auf die sich der Helmstedter Professor bezieht, die sich heute jedoch nicht mehr nachweisen lassen.

Trew bekleidet seit 1744 das Amt des Director Ephemeridum der Deutschen Akademie der Naturforscher (Leopoldina). In dieser Funktion gibt er das Journal der Vereinigung heraus, was ihm eine wichtige Rolle als medizinischem Fachredakteur zuweist ${ }^{91}$. In den späten 1740er Jahren macht er zudem mit der Publikation illustrierter Pflanzenwerke auf sich aufmerksam. Heister seinerseits geniesst nach wie vor einen hervorragenden Ruf als wissenschaftlicher Autor, akademischer Lehrer und praktisch tätiger Arzt und Chirurg.

Die Korrespondenten setzen ihren Gedankenaustausch in dem gewohnt vertraulichen Briefton und in der gesamten bislang entwickelten Themenbreite fort. Die grosse Briefdichte dieser Jahre ist auf eine Verlagerung des Korrespondenzschwerpunkts von fachlichen zu privaten Inhalten zurückzuführen, welche vorrangig um zwei familiäre Belange der Familie Heister kreisen: den Tod der Schwiegermutter und die Heirat der ältesten Tochter. Trew meldet am 2. August 1747 nach Helmstedt:

Nachdeme ich gestern, als den 1 . August Abends vernommen, daß am verwichenen Sonnabend, als den 29 Julii, die Frau SchwiegerMutter [...] mit Todt abgegangen, so habe in erinnerung der ehemaligen erhaltenen Ordre und annoch in Handen habenden Schriften nicht unterlassen sollen, davon alsobalden die Nachricht zu ertheilen und wie ich mich weiters dabey zu verhalten habe um weitern befehl zu bitten, zumalen das Blanquet auf den Wolseeligen Herrn Dr. Wiedmann ausgestellet ist und ich ein anderes bisher nicht erhalten habe.

89 Heister 71, 28.6.1743.

90 Trew 332, 9.5.1744.

91 Siehe dazu Schnalke 2002. 
Damit aber bis zu Erlangung einer Antwort ich nichts versäumen möge, hab ich mich desfalls bey Herrn Dr. Rinder erkundiget, und vernommen, daß ich gar wohl eine beliebige Ordre erwarten könnte. ${ }^{92}$

Heister reagiert auf diese Nachricht sofort. Ihm ist viel daran gelegen, die ihm zustehenden 650 Gulden so schnell wie möglich zu bekommen. Er überschickt Trew eine persönliche Vollmacht, bittet ihn, das Geld über Widmann II an ihn weiterzuleiten und dafür Sorge zu tragen, dass bei der Testamentseröffnung ein Jurist, der Heisters Interessen vertritt, zugegen ist ${ }^{93}$. Trew leistet diesem Wunsch Folge. Er sieht sich jedoch genötigt, Heister mitzuteilen, dass das Geld nicht so leicht zu bekommen und dass dieser keineswegs von seiner Schwiegermutter testamentarisch bedacht worden sei ${ }^{94}$. Heister reagiert bestürzt. Er beklagt die Hartherzigkeit seiner Schwiegermutter ${ }^{95}$, versichert sich in Helmstedt eines Rechtsbeistands ${ }^{96}$ und beauftragt den Nürnberger Juristen Christoph Adam Rinder (1688-1766) ${ }^{97}$, sich der Sache vor Ort anzunehmen ${ }^{98}$. Trew bittet er, ihn nach bestem Vermögen zu unterstützen und ihn über den jeweiligen Stand der Dinge zu unterrichten ${ }^{99}$. Der Nürnberger kommt den Wünschen Heisters zunächst nicht im erhofften Umfang nach ${ }^{100}$. Als sich die Angelegenheit verzögert, spielt Heister mit dem Gedanken, gegebenenfalls persönlich nach Nürnberg zu reisen, um den Fall selbst in die Hand zu nehmen ${ }^{101}$. Der Rechtsstreit wird jedoch letztlich ohne Heisters direktes Eingreifen geschlichtet, so dass Trew am 3. Februar 1750 nach Helmstedt schreiben kann: «[...] habe Euer Wohlgebohren Nachricht zu geben, daß wegen der ehemals mir committirten [...] Schuld forderung noch eine carta bianca in meinen Händen ist: ob ich nun solche Dero Frau Tochter und Herrn Eydam extradiren, oder in ihrem bey seyn zerreissen oder was ich sonsten damit machen soll, erwarte befehl.»102

Das zweite grosse private Briefthema dieser Korrespondenzphase handelt von der anstehenden Vermählung Margaretha Katharina Justina Heisters mit Widmann II, eben jenem Medizinstudenten, den Heister als Kostgänger

92 Trew 333, 2.8.1747.

93 Heister 75, 11.8.1747.

94 Heister 76, 17.9.1747.

95 Heister 76, 17.9.1747.

96 Heister 78, 19.12.1747.

97 Rinder war seit 1715 als Advokat in Nürnberg tätig; vgl. Schmidt-Herrling 1940, 498.

98 Heister 75, 11.8.1747.

99 Heister 78, 19.12.1747; Heister 82, 21.5.1748; Heister 83, 16.7.1748; Heister 85, nach 19.9.1748.

100 Als Heister von Trew endlich den gewünschten, offenbar ausführlichen Bericht erhielt, antwortet er: «Hätte ich von diesem eher was Nachricht deßhalben gehabt, hätte ich mich zu ruhe und gedult gar gern gegeben, und es nicht so negiret.» Heister 81, 23.4.1748.

101 Heister 83, 16.7.1748.

102 Trew 334, 3.2.1750. 
in sein Haus aufgenommen hatte. Widmann hat sich nach seiner Promotion mit Margaretha Heister verlobt und ist danach zu einer längeren Studienreise aufgebrochen. Im Verlauf des Frühjahrs oder Sommers 1747 trifft der junge Arzt wieder in seiner Heimatstadt Nürnberg ein, sucht - entgegen seinen Versprechungen - aber seine Braut in Helmstedt nicht auf. Voll Sorge wendet sich Heister am 17. September 1747 an Trew mit der Bitte, ihm «im Vertrauen einige Nachricht von Herrn Dr. Widman zu geben, wo Er jetzo seye, was er mache, ob Er vielleicht verreist oder unbäßlich seye» ${ }^{103}$. Zwei Monate später lässt er die Frage folgen, ob Widmann, der «was langsam» schreibe, inzwischen ins Nürnberger Collegium medicum aufgenommen worden sei ${ }^{104}$. Schliesslich legt er Trew in seinem Brief vom 19. Dezember 1747 die Gründe seines Nachfragens dar:

Ich zweifele auch nicht, daß Ihnen werde bekannt seyn, daß Er [Widmann] Sich, da Er das Jahr nach seiner Promotion exprés wieder hierher gekommen, sich mit meiner Jüngsten Tochter gantz solenn und ordentlich in praesentia verschiedener Professoren verlobet hat, da ich von andern vernommen, daß gantz Nürnberg davon voll seyn solle, auch nunmehr über 2 Jahre von seinen reißen sehr offt an mich und seine Braut geschrieben, als Bräutigam an Braut und Schwieger Vatter zu schreiben pflegen, solches auch noch zu verschiedenen mahlen von Nürnberg seit seiner wiederkunft von reißen gethan und Sie seiner Treue und beständigen Liebe versichert, auch vermeldet, daß Er bald nach Laurentzi kommen wolle um Sie abzuhohlen, wie ich Ihnen schon einmal geschrieben. Nachdem aber, ongeachtet wir Ihm allezeit prompt und hofflich geantwortet haben, hat Er langsammer und kaltsinnlicher geschrieben, und ongeachtet wir beide Ihm auch noch im october, da seyn Geburts gewesen, darzu gratulirt, haben aber hierauf bißdato keine Antwort erhalten, daher uns allerlei Zweifel und sorge deßhalben entstehet, ob Er unbäßlich oder sonsten was anderes ursache daran seye, Ich habe schon alle Ausstattungskosten, da Er geschrieben hatte, gleich nach Laurentzi zu kommen, aufgewannt welches ich Ihm auch geschrieben, weiß also nicht was die Hinderung daran seyn möge..$^{105}$

Heister kann Widmanns Verhalten nicht billigen und befürchtet, dass dadurch die Ehre seiner Familie in Schimpf und Schande gezogen werde ${ }^{106}$. Zunächst bittet er seinen Nürnberger Korrespondenten lediglich, weiterhin vertrauliche Erkundigungen einzuholen und ihn regelmässig zu unterrichten ${ }^{107}$. Schliesslich ersucht er ihn jedoch, aktiv einzugreifen und Widmann in seinem Namen ins Gewissen zu reden ${ }^{108}$.

103 Heister 76, 17.9.1747.

104 Heister 77, 21.11.1747.

105 Heister 78, 19.12.1747.

106 Heister 78, 19.12.1747; Heister 79, 16.2.1748.

107 Heister 78, 19.12.1747. - Heister bittet Trew, «diese beschimpfung von mir und meinem hause helfen abzuwenden [...] und doch solches alles sub rosa zu tractiren.» Heister 79, 16.2.1748.

108 «...] also will Dieselbe gantz ergebenst bitten, Sich zu bestreben Ihn in diesem guten Sinn und Vorsatz zu erhalten, und anzutreiben [...]» Heister 82, 21.5.1748; vgl. auch Heister 83, 16.7.1748; Heister 84, 20.8.1748. 
Heisters Hilferufe werden immer drängender. Er erinnert Trew an die vielfältige Unterstützung, die er ihm stets habe zuteil werden lassen ${ }^{109}$. Mit Erleichterung reagiert er auf ein Schreiben Trews, in dem ihn dieser über ein Gespräch mit Widmann informiert und seine weitere Unterstützung zusagt ${ }^{110}$. Heister verdeutlicht daraufhin dem Nürnberger Vertrauten nochmals seine Position. Er habe Widmann klare Worte geschrieben, ihn zur Besinnung gerufen und werde nun, da sein künftiger Schwiegersohn ihm so lange nicht geantwortet habe, keinen weiteren Brief mehr an diesen richten $^{111}$. Das daraufhin von Widmann erhaltene Schreiben, in welchem dieser seine «unbäslichkeit» für sein Verhalten verantwortlich macht und sein baldiges Kommen ankündigt, führt Heister auf Trews entschlossene «Interposition und Zureden» zurück ${ }^{112}$. Er bedankt sich bei dem Nürnberger und bittet ihn, Widmann in seinem guten Vorsatz «zu erhalten, und anzutreiben ${ }^{113}$, um einem erneuten Sinneswandel vorzubeugen. Seinem Schreiben legt Heister einen Brief an seinen künftigen Schwiegersohn bei, den Trew mit entsprechendem Nachdruck überreichen soll. Als Widmann erneut mehrere Wochen verstreichen lässt, reagiert Heister bitter und gereizt ${ }^{114}$. Er möchte die Angelegenheit endlich zu einem Abschluss bringen und droht schliesslich: «Sollte Herr Dr.Widmann uns nicht bald die Ehre geben zu uns zu kommen, so werde ich [...] Selbsten eine Reiße hienauf thun müßen, und sehen ob ich selbige [Sache] alsdann nicht möge können zum stande bringen.» ${ }^{115}$

Das Blatt wendet sich, als Widmann Heister mitteilen kann, dass er inzwischen alle Voraussetzungen für eine Aufnahme in die Nürnberger Ärzteschaft erfüllt ${ }^{116}$. Heister vermutet, dass Trew dem jungen Widmann hierbei den Weg geebnet hat, und bittet den Nürnberger Briefpartner, nun auch die tatsächliche Aufnahme seines künftigen Schwiegersohns ins Collegium medicum voranzutreiben und diesen endgültig zur Reise nach Helmstedt zu

109 Heister berief sich auf die Zeiten «unserer alten und langen Freundschafft» und bittet um Trews Unterstützung für sich, «als Ihren alten ehrlichen und aufrichtigen Praeceptori, der Ihnen auch vor diesem, ohne ruhm zu melden, viele gute aufrichtige Dienste gethan [...]». Heister 79, 16.2.1748.

110 Heister 81, 23.4.1748.

111 Heister 81, 23.4.1748.

112 Heister 82, 21.5.1748. - Widmanns «unbäslichkeit» dürfte mit einem Krankheitsgeschehen in Zusammenhang gestanden sein, über welches die Korrespondenz zwischen Widmann und Trew aus dem Jahr 1746 Auskunft gibt; Widmann II 4, 15.2.1746; Widmann II 5, 5.10.1746; Trew 818, nach 5.10.1746; Widmann II 6, 5.11.1746.

113 Heister 82, 21.5.1748.

114 Heister 83, 16.7.1748.

115 Heister 83, 16.7.1748.

116 Heister habe mit Vergnügen vernommen, so schrieb er an Trew, «daß endlich des Herrn Dr. Widmanns Specimen anatomicum und practicum abgelegt seye [...]». Heister 85, nach 19.9.1748. 
bewegen ${ }^{117}$. Widmann bricht schliesslich nach Helmstedt auf. Die Ehe wird geschlossen, die Affäre löst sich zur allgemeinen Zufriedenheit. Heister nennt Widmann in seinem Schreiben vom 19. November 1748 seinen «neuen Herrn Schwieger Sohn» und dankt Trew

auch für die viele mühe, welche ich Ihnen durch Antreibung und beföderung der hierher Reise deßelben sich gegeben haben, welche sich sonsten ohne Sie wohl noch gar sehr lang mögte trainirt, und uns also immer in Sorgen und ungewißheit mögte gelaßen haben, wovon wir aber dadurch endlich sind befreyet worden, Sie lieben nun einander recht sehr, und verhoffe ich davon alles gutes und Freude in meinem Alter an Ihnen zu erleben, will Sie auch beide zu beständiger wohlgewogenheit Ihnen bestens hiermit empfehlen. ${ }^{118}$

Stehen diese familiären Themen auch im Zentrum dieser Korrespondenzphase, so geraten fachliche Inhalte nie aus dem Blickfeld der Briefpartner. Trew bleibt für Heister der botanische Lieferant und Diskutant. Zunehmend wichtiger wird er jedoch als Sammler von Pflanzenbildern und pflanzenkundlicher Literatur. Trew übernimmt im wissenschaftlichen Austausch gegenüber Heister die Rolle eines Quellenarchäologen, -kollektors und -archivars, der seine Schätze nicht nur selbst in illustrierten Editionen «dem publico» vorzulegen beginnt, sondern diese auch für wissenschaftliche Anfragen seines Korrespondenten bereitstellt. So wird er von Heister gebeten, für ihn das «Epitome Matthioli von Joachim Camerario» ${ }^{119}$ entweder «um billigen prei ${ }_{{ }^{120}} \mathrm{zu}^{\mathrm{z}}$ erwerben oder ihm sein eigenes Exemplar leihweise zu überlassen ${ }^{121}$. Zunächst äussert sich Heister nur vage, wozu er das Werk benötigt:

ich habe es zu was das ich unter händen zu ediren, nöthig. Ich mögte auch wohl wißen was sie für editiones von eben dieses Camerarii Herbario haben. Ich habe eine sehr schöne teutsche von 1586 mit schönen illuminirten figuren durch und durch worinnen wohl viele von Gesnero, aber auch doch viele sein eigen seyn mögen. ${ }^{122}$

Trew lässt sein Exemplar durch Widmann an Heister übermitteln, der es nach Durchsicht wieder zurückreicht und seine Anfrage präzisiert. Vor allem geht es ihm um ein Urteil Trews über die Qualität der Abbildungen sowie darum, ob man unterscheiden könne, welche Illustrationen von Gesnerus und welche von Camerarius in Auftrag gegeben worden sind. Dahinter steht Heisters Absicht, in einer Veröffentlichung «dem Camerario was mehr Ehre inter scriptores, welche zum Methodo botanices gelegenheit gegeben,

117 Heister 85, nach 19.9.1748.

118 Heister 87, 19.11.1748.

119 Camerarius, Joachim, De Plantis Epitome utilissima Petri Andreae Matthioli Senensis [...] (Frankfurt am Main 1586).

120 Heister 83, 16.7.1748.

121 Heister 85, nach 19.9.1748.

122 Heister 85, nach 19.9.1748. 
zuwegen [zu] bringen [...], als Er bißher gehabt [...]» ${ }^{123}$. Trew verfasst daraufhin eine Abhandlung, die er so einrichtet, «daß ich sie zugleich zu einer Vorrede gebrauchen kan, ohne viele Veränderung und neue Arbeit [...]» ${ }^{124}$. Offenbar bleiben darin für Heister einige Fragen offen, denn er nutzt 1750 «eine kleine Spazierreise auf Nürnberg auf ein paar Wochen» ${ }^{125}$, zu der ihn sein Schwiegersohn Widmann aufgefordert hat, um seine Studien in der Trewschen Bibliothek weiterzuführen. Wieder nach Helmstedt zurückgekehrt, dankt er dem Nürnberger

hiermit noch gantz ergebenst wegen der vielen Ehre die Sie mir Zeit meines Auffenthaltens in Nürnberg erwiesen, und bitte um Vergebung daß ich Ihnen so viele Mühe wegen durchsehung der Gesnerischen und Camerarischen gemahlten Pflantzen, als auch noch so vielen andern sehr schönen gemahlten Pflantzen und Durchsehung so vieler raren und kostbaren botanischen wercken, an welchen allen ich sehr groses Vergnügen empfunden [gemacht habe]. ${ }^{126}$

Die weitere medizinische Korrespondenz zeigt Trew vor allem als wissenschaftlichen Mediator. Heister unterstreicht dessen Bedeutung als Director Ephemeridum, dem er Beiträge dritter Personen anempfiehlt ${ }^{127}$ und eigene Beobachtungen sowie Observationes seines verstorbenen Schwiegersohns Crell zuschickt ${ }^{128}$. So reicht er unter anderem den bereits 1746 für das Commercium litterarium vorgesehenen Extrakt seiner Arbeit über das celsische Lithotomieverfahren ein ${ }^{129}$, berichtet von einer geglückten Steinoperation ${ }^{130}$, einem «niemals gesehenen besondern situ hepatis» ${ }^{131}$, einem «doppeln [!] Magen» und von interessanten Sektionsbefunden an einem «schon vor vielen Jahren hier secirten famoso gesticulatore» ${ }^{132}$.

\section{Anerkennung der pragmatischen Autorität Heisters (1753-1757)}

Die letzte Phase der Korrespondenz umfasst die Jahre 1753 bis 1757. Während Trew trotz seiner ausgedehnten Praxis in Nürnberg und seinen häufigen Konsultationsreisen nach Ansbach die Herausgabe seiner illustrierten Pflanzenwerke vorantreibt, bleibt Heister auch im hohen Alter in seinen akademischen Ämtern und Würden sowie als praktischer Arzt tätig.

123 Heister 87, 19.11.1748.

124 Trew 334, 3.2.1750.

125 Heister 101, vor 21.7.1750.

126 Heister 88, nach 9.10.1750.

127 Heister 101, vor 21.7.1750.

128 Heister 82, 21.5.1748; Heister 90, 29.1.1751; Heister 91, 26.3.1751; Heister 92, 21.5.1751.

129 Heister 90, 29.1.1751.

130 Heister 82, 21.5.1748.

131 Dieses und das folgende Zitat aus: Heister 90, 29.1.1751.

132 Heister 91, 26.3.1751. 
Der Brieffluss erreicht zwar nicht mehr die vorangegangene Dichte ${ }^{133}$, jedoch beherrschen die bislang entwickelten Strukturen und Gegenstände auch weiterhin den Gedankenaustausch. Trew ist für Heister der persönliche Vertraute, dem er immer wieder familiäre Angelegenheiten mitteilt ${ }^{134}$. Daneben sieht Heister in ihm den literarisch produktiven Wissenschaftler, dessen Werken er Beifall spendet ${ }^{135}$ und den er zur Fortsetzung seiner Arbeiten ermuntert, auch wenn dieser zunehmend an die Grenzen seiner Leistungsfähigkeit stösst ${ }^{136}$. Heister bedient Trew als Sammler mit seinen botanischen Schriften ${ }^{137}$ sowie den medizinischen und physikalischen Dissertationen und Programmschriften, die an der Universität Helmstedt erarbeitet werden ${ }^{138}$. Vor allem ist Trew für ihn in dieser Spätphase der Korrespondenz der Wissensmediator, der zum einen durch sein Amt als Director Ephemeridum eine wichtige Rolle im internationalen Wissenstransfer spielt und zum anderen durch seine Kontakte zu Verlegern wissenschaftlicher Werke und zu Illustratoren im süddeutschen Raum eine besondere Bedeutung besitzt ${ }^{139}$.

Neben seinen botanischen Beiträgen bietet Heister auch «etliche besondere wahrnehmung und wichtige curen nebst operationem» ${ }^{140}$ für eine Veröffentlichung in den Ephemeriden an. Hierbei kommt es ihm besonders auf den therapeutischen Aspekt an. Zwar hätten schon andere Autoren von solchen Befunden, etwa einer übergrossen Nase oder der besonderen Art von Hernien, berichtet, zur Behandlung hätten sie sich jedoch nicht geäussert. Heister legt gerade auf diese Seite der ärztlichen Heilkunst sein Augenmerk: «Ich aber habe meine beide [Hernien] operirt und sonderlich

133 Drei Schreiben Trews stehen sieben Briefe Heisters gegenüber, worunter sich ein Briefauszug (Heister 102, 15.6.1753) und eine gedruckte Todesanzeige (Heister 96, 14.5.1756) befinden.Am 6.4.1753 erwähnte Heister eine Zusendung illustrierter Pflanzenwerke, zu der sich kein Anschreiben im Entwurf oder Original erhalten hat; Heister 95.

134 Heister setzte Trew über den Tod seiner ältesten Tochter in Kenntnis, worauf ihm Trew seine lebenslange Verbundenheit ausdrückt; Heister 96, 14.5.1756; Trew 336, 7.6.1756.

135 Heister 95, 6.4.1753; Heister 97, 2.8.1756.

136 Trew 338, 16.11.1756. - Vgl. hierzu die Trewschen Prachtflorilegien Trew 1750-1773, $1750-1786$ und $1763-1784$.

137 Heister 97, 2.8.1756; Trew 338, 16.11.1756; Heister 98, 7.1.1757.

138 Trew 338, 16.11.1756.

139 Heister teilte Trew in seinem Schreiben vom 2.8.1756 seine «neue disposition und Eintheilung 1. der Aloen 2. der Bulbosarum, 3. der Labiatarum, 4. der Capitatarum und 5. der Malvacearum» mit (Heister 97). Da er für diese systematische Arbeit bislang keinen Verleger gefunden hatte, bat er Trew, den Beitrag über die Aloen in die Anhänge der Actorum Naturae Curiosorum einrücken zu lassen. Als ihm Trew nach Rücksprache mit dem Akademiepräsidenten Andreas Elias Büchner (1701-1769) eine Zusage machen konnte (Trew 338, 16.11.1756), reichte Heister seine Arbeit und seine Illustrationen ein, Heister 98, 7.1.1757.

140 Dieses und das folgende Zitat aus Heister 98, 7.1.1757. 
untersucht was in dem so greulich grosen Bruch gewesen.» Durch eine Veröffentlichung dieser Beobachtungen will er die operative Therapie zum wissenschaftlichen Verhandlungsgegenstand machen. Trew soll ihm für die damit verbundene Aufwertung der Chirurgie publizistische Hilfestellung leisten.

Überraschend beleuchtet die Endphase der Korrespondenz schliesslich noch die praktische Seite des ärztlichen Alltags von Christoph Jacob Trew. Sie zeigt den Nürnberger in seinem diagnostischen und therapeutischen Handeln sowie in seinem Verhältnis zur pragmatischen Autorität Heisters. Trew legt Heister in einem Schreiben vom 6. Juni 1755 ausführlich die Krankengeschichte eines «Herrn von Holtzschuer» aus Nürnberg dar ${ }^{141}$, der an einer Geschwulst unter und vor dem rechten Ohr leidet. Er zeigt sich dabei als Arzt, der gewissenhaft die Anamnese erfragt, den Befund erhebt, eine Diagnose stellt und ein differenziertes Therapieregime entwickelt, das er dem jeweiligen, genau protokollierten Krankheitsverlauf anpasst.

Als Trew im September 1754 zu Rate gezogen wird, erfährt er aus der Vorgeschichte, dass sich ähnliche Gewächse, die in den Jahren 1752 und 1753 an der gleichen Körperregion aufgetreten sind, von selbst zurückgebildet haben. Trew diagnostiziert das Leiden als «verhärtete Kieferdrüse» (Glandula maxillaris indurata), entstanden infolge einer «Verstopfung durch sehr zähen Schleim» (infarctus tenacioris pituitae). Der Tumor hat die Grösse eines Apfels, ist beweglich, jedoch gegen die knöcherne Unterlage nicht verschieblich. Noch verspürt der Patient keinen Schmerz in der Geschwulst. Trews Therapiekonzept zielt auf eine Konsistenzänderung und Ableitung der Schleimmassen, eine Blutreinigung, eine Erweichung und «Zertheilung» des Tumors sowie auf eine Laxation des Magen-Darm-Traktes. Hierzu verordnet er verschiedene innerlich und äusserlich wirkende Arzneien und beurteilt den Behandlungsverlauf nach Wachstum, Form-, Farb- und Konsistenzänderung sowie nach Schmerzhaftigkeit des Tumors. Sowohl für die Zusammenstellung der äusserlichen Medikation als auch in der Verlaufskontrolle arbeitet er mit einem Chirurgen zusammen. Nach jeder Visite passt er die Dosierung und Zusammensetzung seiner Arzneien dem aktuellen Befund an, ohne von den einmal festgelegten Therapiepfaden abzuweichen.

Trews Behandlung spricht nicht an. Ab März 1755 nimmt der Tumor rasch an Grösse zu, worauf der Nürnberger Arzt die schleimabführende und blutreinigende Therapie zeitweise aussetzt. Ende des Monats tritt erstmalig «ein Stechen in demselben [auf], und zeigten sich auch hin und wieder, sonderlich um die Mitte, einige rothe Flecken». Trew befürchtet eine Exulzeration des

141 Sämtliche Textbelege zum Fall Holzschuher stammen aus Trew 335, 6.6.1755. 
Tumors und verordnet ein schmerz- und entzündungshemmendes Pflaster. Die Geschwulst wuchs daraufhin zwar langsamer, doch Mitte April 1755 entdeckt Trew im Mund seines Patienten eine knotige Auftreibung. Er muss gestehen, dass «bey so langem Gebrauch der bisher ganz ordentlich continuierten medicamenten die geringste Hilfe nicht verspühret wurde [...]». Weder ein von Dritten empfohlenes «Specificum» aus den Blättern der Gründwurtz noch das Ende Mai 1755 im vollen Umfang wieder aufgenommene ursprüngliche Therapieregime bringen eine Besserung. Im Gegenteil, neben der Beeinträchtigung durch den ausgedehnten Tumor verschlechtert sich nun auch das Allgemeinbefinden des Kranken. Er leidet unter katarrhalischen Erscheinungen mit Sprech- und Schluckbeschwerden und unter Schwächeanfällen. Als am 3. Juni 1755 der «tumor vom chirurgo mit starckem betasten genau examiniret wurde, verfiel der Herr Patient abermals nicht nur alleine in eine noch stärckere Ohnmacht, sondern es brachen auch so gar einige motus convulsivi aus [...]». An den darauffolgenden Tagen plagen Übelkeit und Erbrechen den Kranken.

Nun wendet sich Trew an Heister: «Was aber ferner zu Hebung dieses so grossen als hartnäckigen übels anzuwenden, und insonderheit, ob die remedia externa magis ad suppurationem quam resolutionem promovendam einzurichten seyn: darüber wird um einen hocherfahrnen Rath hiemit inständigst gebetten.» Trew steht in einem therapeutischen Dilemma. Die interne Medikation hat versagt. Die gemeinsam mit dem Chirurgen entwickelten und durchgeführten externen Massnahmen haben ebensowenig Erfolg gebracht wie die laienmedizinische Kur. Dagegen sieht sich Trew gegenüber Heister genötigt, zu zwei bislang unterbliebenen Behandlungsmöglichkeiten Stellung zu nehmen. Zum einen habe er auf den Einsatz von Quecksilberpräparaten verzichtet, weil er «bey tumoribus scirrhosis glandularum salivalium davon mehr Schaden als Nuzen angemerket habe». Zum anderen ist es für ein chirurgisches Vorgehen, das im Frühstadium der Erkrankung die Therapie der Wahl gewesen wäre, inzwischen zu spät. Trew entschuldigt sein Versäumnis damit,

daß dießer tumor nicht nur alleine im Anfang vagus gewesen, sondern auch zum zweyten mal sich von selbst wieder verzogen hat, man also auch solches desto eher das drittemal hoffen kunnte; daß, als solcher keine Veränderung mehr zeigte, die harte wintersZeit ein fiele, und, da er zu wachsen anfinge, solches Wachsthum so schnell überhand nahme, daß man an eine operation nicht mehr gedencken kunnte.

Jetzt sei der Tumor so angewachsen, dass eine Operation eine grosse Lebensgefahr darstelle und für ein solches Vorgehen auch «nicht allezeit ein chirurgus beyhanden ist, der dazu so wohl geschickt und erfahren als auch beherzt genug ist». 
Heister bestätigt in dem Entwurf seines schriftlichen Konsils das bisherige Vorgehen Trews ${ }^{142}$ und gibt für die einzelnen Therapieziele alternative Rezepturen an, die sich in seiner Praxis bewährt haben. Darüber hinaus schlägt er als Adjuvantien zur «Stärckung des Magens, Hertzens und Kopffs» vor, bei der Mahlzeit «von einem guten alten ungarischen und insonderheit Tockayerwein zu geniesen, auch bei gutem temperiertem wetter mit gelindem Spatzieren fahren sich eine mässige Bewegung zu weilen zu machen [...]». Im Postskriptum äussert sich Heister zur Möglichkeit eines chirurgischen Vorgehens:

Ob Sich diese Geschwulst, wenn sie etwa mit Artzeneyen sich nicht wolte zertheilen laßen, noch durch eine chirurgische operation möge können weg gebracht werden, gleichwie ich verschiedene solche am Hals dadurch wieder glücklich curiret habe, läßet sich ohne dieselbe gesehen zu haben nicht bestimmen.

Hier kommt die «Medizin im Brief» offensichtlich an ihre Grenzen. Die Klärung der Operationsindikation kann ebensowenig wie die Durchführung der chirurgischen Behandlung selbst von dem operativ nicht versierten Briefpartner vor Ort übernommen werden ${ }^{143}$. Die Kompetenz liegt hier ausschliesslich bei Heister. Damit weist er auf ein Defizit hin, das Trew mit den meisten seiner Berufskollegen in jener Zeit teilt und das Heister für sich beseitigt hat - die Ausblendung der chirurgischen Dimension des eigenen ärztlichen Handelns. Während Trew in seinem therapeutischen Vorgehen noch weitgehend den internistischen Typus des Arztes verkörpert, der zwar die Wichtigkeit der externen Behandlung erkennt, sie jedoch selbst nicht ausübt und hierfür mit einem Chirurgen zusammenarbeitet, vereint Heister beide Medizinen in einer Person. Darin bleibt Heister seinem einstigen Schüler stets voraus.

Bei aller persönlichen Vertrautheit und fachlichen Gleichrangigkeit, die sich im Briefverhältnis zwischen Trew und Heister eingestellt haben, zeigt somit der Abschluss der Korrespondenz eine deutliche Asymmetrie auf der Ebene der medizinischen Praxis. Trew ist trotz der grossen Erfahrung, die er in langjähriger ärztlicher Tätigkeit gewonnen hat, im Berufsbild seiner Zeit verhaftet und stösst damit notwendigerweise in manchen Fällen an seine therapeutischen Grenzen. Auf diesem Gebiet bleibt er lebenslang der Schüler, der sich ratsuchend an Heister als seine medizinisch-pragmatische Autorität wendet.

142 Heister entwarf sein Konsil unter dem Datum vom 16.6.1755 auf den leer gebliebenen Bogen der Trewschen Krankengeschichte. Es wird unter der Signatur der Historia morbi Trews aufgeführt: Trew 335, 6.6.1755. - Heister bestätigte Trew, er habe äusserlich wie innerlich «lauter sehr herrliche und kräftige Mittel verordnet und gebrauchen laßen», und zwar die «kräftigsten abfürenden zertheilenden und corrigirenden Artzneyen».

$143 \mathrm{Zu}$ den Möglichkeiten und Grenzen einer «Chirurgie per Post» siehe ausführlicher Ruisinger 2005. 


\section{Resümee}

Überblickt man die Entwicklung des Briefverhältnisses zwischen Heister und Trew, lässt sich folgendes Grundmuster erkennen: Die Basis für ihre enge berufliche und persönliche Bindung legten die späteren Briefpartner mit ihrem direkten, vertrauten Umgang als Lehrer und Schüler an der Universität Altdorf. Dieses hierarchische Verhältnis gab zunächst auch die Bahnen vor, in denen der Briefwechsel zu Zeiten der Studienreise Trews eingerichtet wurde. Heister, der infolge seiner Lehrverpflichtung an Altdorf gebunden war, erwartete gewissermassen als Gegenleistung für seine Ausbildungsinvestition eine Reihe wissenschaftlicher Dienste. Dabei wollte er vor allem von den fachlichen Kontakten und Beobachtungen sowie von den Zugriffsmöglichkeiten auf die aktuelle Literatur profitieren, die sich für Trew auf der Peregrinatio academica ergaben. Erst als sich der junge Medikus in Nürnberg niederliess und dort sowohl als ärztlicher Praktiker wie auch als Naturforscher die ihm offenstehenden beruflichen Rollen produktiv auszufüllen begann, setzte ein Emanzipationsprozess aus dem bisherigen Patronageverhältnis ein. Aus dem Lehrer-Schüler-Dialog wurde ein Austausch zwischen Briefpartnern, die in fast allen Belangen gleichberechtigt waren, die aber sehr unterschiedlichen Wirksphären zugehörten. Damit spiegelt ihr Briefwechsel - und das unterscheidet ihn von vielen anderen gelehrten Korrespondenzen - auch das Verhältnis von akademisch-universitärer und wissenschaftlich ambitionierter urbaner Medizin wider.

Wichtig für diesen Prozess war, dass der ehemalige Student seinem einstigen Lehrer spezifische Angebote machen konnte, die diesem offenbar aus anderer Richtung in entsprechender Qualität, Quantität und Praktikabilität so nicht offenstanden. Gerne nahm der Helmstedter die Nürnberger Offerten an und sah sich aufgefordert, im Gegenzug dem fachlichen Ansinnen des Briefpartners mit seinen Möglichkeiten gerecht zu werden. Als gleichrangige Fachgelehrte trafen sich Heister und Trew insbesondere auf dem Gebiet der Botanik, das in jener Zeit zwar mit dem Ziel einer grundlegenden Systematisierung und Klassifizierung intensiv bearbeitet wurde, das gleichzeitig aber in seiner wissenschaftlichen Formung noch ein weitgehend offenes Terrain war und noch vergleichsweise wenig allgemein akzeptierte autoritative Setzungen aufwies. Mit zunehmender Dauer der Korrespondenz festigten private und vertraulich-persönliche Themen die Brieffreundschaft. Allerdings offenbart das Briefverhältnis zwischen Heister und Trew schliesslich in jenem Bereich ein Ungleichgewicht, der für beide Gelehrte das Zentrum ihrer beruflichen Existenz ausmachte - in der ärztlichen Praxis. Während sich Lehrer und Schüler in ihren internistischen Überlegungen und Emp- 
fehlungen gegenseitig respektierten und bestärkten, blieb Heister als praktizierender Chirurg gegenüber Trew ein Experte auf operativem Gebiet und daher letztlich der «komplettere» Arzt.

Die Korrespondenz erfuhr somit ihre Dynamik aus dem Umstand, dass die Briefpartner neben breit entwickelten Feldern eines gleichrangigen fruchtbaren Gebens und Nehmens auch Bereiche für sich reklamieren konnten, auf welchen sie dem Kollegen gegenüber einen Vorsprung hatten. Trew war und blieb für Heister als Sammler und Wissensmediator interessant, Heister figurierte bis zum Schluss in Trews Augen als zentrale pragmatische Autorität in Fällen, in denen ein chirurgisches Eingreifen zur Diskussion stand.

\section{Literatur}

Barras,Vincent/Micheline Louis-Courvoisier (éds), La Médecine des Lumières. Tout Autour de Tissot, Bibliothèque d'Histoire des Sciences (Genf 2001)

- /Martin Dinges (Hrsg.), Krankheit in Briefen, Medizin, Gesellschaft und Geschichte, Beihefte (Stuttgart 2005, im Druck)

Boschung, Urs, «Iatromechanik und Chirurgie bei Lorenz Heister», Gesnerus 40 (1983) 31-41

Brockliss, Laurence, "Consultation by Letter in Early Eighteenth-Century Paris: The Medical Practice of Etienne-François Geoffroy", in: Ann La Berg/Mordechai Feingold (eds), French Medical Culture in the Nineteenth Century, The Wellcome Institute Series in the History of Medicine (Amsterdam/Atlanta 1994) 79-117

Brucker, Jacob/Johann Jacob Haid (Hrsg.), Bilder-sal heutiges Tages lebender, und durch Gelahrtheit berühmter Schrifft-steller, 20 Zehend in 2 Bänden (Augsburg 1741-1755)

Forster, Elborg, "From the patient's point of view. Illness and health in the letters of Liselotte von der Pfalz (1652-1722)", Bulletin of the History of Medicine 60 (1986) 297-320

Gehrke, Christian, Die Patientenbriefe der Mathilde von Berenhorst (1808-1874). Edition und Kommentar einer Krankengeschichte von 1832-1833 (Diss. med. Göttingen 2000)

Geiger, Alois, Lorenz Heister als Geburtshelfer und gynäkologischer Chirurg. Vergleichende Untersuchung anhand der verschiedenen Auflagen seiner «Chirurgie», Zürcher medizingeschichtliche Abhandlungen, Neue Reihe, 162 (Zürich 1983)

Gosteli, Leo/Urs Boschung/Peter Brosche, Astronom, Weltbürger, Blasensteinpatient. Franz Xaver von Zachs Briefe an Rudolf Abraham von Schiferli 1821-1832, Gesnerus Supplementum 45 (Basel 1998)

Heister, Lorenz, De Cataracta, Glaucomate et Amaurosi Tractatio [...] (Altdorf 1713)

- Apologia et uberior illustratio systematis sui de cataracta, glaucomate et amaurosi [...] (Altdorf 1717a)

- Compendium anatomicum [...] (Altdorf 1717b)

- Chirurgie [...] (Nürnberg 1719a)

- Compendium institutionum sive fundamentorum medicinae (Helmstedt 1736)

- Practisches Medicinisches Handbuch [...] (Leipzig 1744)

- Johannes Wilhelm Ilsemann, Dissertatio chirurgica de Lithotomiae Celsianae praestantia et usu (Helmstedt 1745)

- Medicinische, Chirurgische und Anatomische Wahrnehmungen (Rostock 1753)

Hörz, Herbert, Physiologie und Kultur in der zweiten Hälfte des 19. Jahrhunderts. Briefe an Hermann von Helmholtz (Marburg 1996)

Kempe, Michael, «Die Anglo-Swiss Connection. Zur Kommunikationskultur der Gelehrtenrepublik in der Frühaufklärung», in: Robert Seidel (Hrsg.), Zur Kommunikationskultur der Gelehrtenrepublik in der Frühaufklärung, Cardanus. Jahrbuch für Wissenschaftsgeschichte 1 (Heidelberg 2000) 17-91 
Korff, Rüdiger, Das Berufsethos in der Chirurgie Lorenz Heisters (1683-1758), Zürcher medizingeschichtliche Abhandlungen, 107 (Zürich 1975)

Leporin, Christian Polycarpo, Ausführlicher Bericht vom Leben und Schrifften des durch ganz Europa berühmten Herrn D. Laurentii Heister [...] (Quedlinburg 1725)

Mann, Gunter, «Medizinisch-naturwissenschaftliche Buchillustration im 18. Jahrhundert in Deutschland», Sitzungsberichte der Gesellschaft zur Beförderung der gesamten Naturwissenschaften zu Marburg 84 (1964) 3-48

Meyer, Jörg, «〈... als wollte mein alter Zufall mich jetzt wieder unterkriegen〉. Die Patientenbriefe an Samuel Hahnemann im Homöopathie-Archiv des Instituts für Geschichte der Medizin in Stuttgart», Jahrbuch des Instituts für Geschichte der Medizin der Robert-BoschStiftung 3 (1986) 63-79

Nissen, Claus, Die botanische Buchillustration. Ihre Geschichte und Bibliographie, 2 Bde. (Stuttgart 1951)

Pirson, Julius, «Der Nürnberger Arzt und Naturforscher Christoph Jakob Trew (1695-1769)», Mitteilungen des Vereins für Geschichte der Stadt Nürnberg 44 (1953) 448-575

Rees, Maximilian, Ueber die Pflege der Botanik in Franken von der Mitte des 16. bis zur Mitte des 19. Jahrhunderts (Erlangen 1884)

Risse, Günter, "Doctor William Cullen, Physician, Edinburgh. A Consultation Practice in the Eighteenth Century", Bulletin of the History of Medicine 48 (1974) 338-351

Rosen, George, "Lorenz Heister on Acupuncture: An Eighteenth Century View", Journal of the History of Medicine and Allied Sciences 30 (1975) 386-388

Ruisinger, Marion Maria, «Auf Messers Schneide. Patientenperspektiven aus der chirurgischen Praxis Lorenz Heisters (1683-1758)», Medizinhistorisches Journal 36 (2001) 309-333

- «Der flüssige Kristall. Anatomische Forschung und therapeutische Praxis bei Lorenz Heister (1683-1758) am Beispiel des Starleidens», in: Jürgen Helm/Karin Stukenbrock (Hrsg.), Anatomie. Sektionen einer medizinischen Wissenschaft im 18. Jahrhundert (Stuttgart 2003) 101-125

- «Der vierte Weg zum Stein. Die Lithotomie nach «Frère Jacques` als Goldstandard bei Johann Jacob Rau und Lorenz Heister», in: Vorstand der Internationalen Nitze-Leiter-Forschungsgesellschaft für Endoskopie (Hrsg.), Lithotomie versus Lithotripsie. Historisch und rezent, Schriften der Internationalen Nitze-Leiter-Forschungsgesellschaft für Endoskopie, 4 (Wien 2004) 37-45

- «Chirurgie im Brief. Das Beispiel der Konsiliarkorrespondenz Lorenz Heisters (16831758)», in:Vincent Barras/Martin Dinges (Hrsg.), Krankheit in Briefen, Medizin, Gesellschaft und Geschichte, Beihefte (Stuttgart 2005, im Druck)

Rusnock, Andrea A., «The Correspondence of James Jurin (1684-1750), Physician and Secretary to the Royal Society», Clio Medica 39 (Amsterdam 1996)

Schlipp, Friedrich: «Laurentius Heister in seiner Bedeutung für die Augenheilkunde», Janus 15 (1910) 372-411

Schmidt-Herrling, Eleonore, Die Briefsammlung des Nürnberger Arztes Christoph Jacob Trew (1695-1769) in der Universitätsbibliothek Erlangen (Erlangen 1940)

Schnalke, Thomas, «Ein Korb für Göttingen. Christoph Jacob Trew im Briefwechsel mit dem Chirurgen Carl Friedrich Gladbach», Medizinhistorisches Journal 29 (1994) 233-275

- Natur im Bild. Anatomie und Botanik in der Sammlung des Nürnberger Arztes Christoph Jacob Trew. Eine Ausstellung aus Anlaß seines 300. Geburtstages, Schriften der Universitätsbibliothek Erlangen-Nürnberg, 27 (Erlangen 1995)

- Medizin im Brief. Der städtische Arzt des 18. Jahrhunderts im Spiegel seiner Korrespondenz (Stuttgart 1997)

- «Die korrespondierende Akademie - Organisation und Entwicklung der Leopoldina um 1750», in: Benno Parthier/Dietrich von Engelhardt (Hrsg.), 350 Jahre Leopoldina - Anspruch und Wirklichkeit. Festschrift der Deutschen Akademie der Naturforscher Leopoldina 16522002 (Halle/Saale 2002) 95-119

Spiess, Ernst, Naturhistorische Bestrebungen Nürnbergs im XVII. und XVIII. Jahrhundert. Leben und Werke ihrer Beschützer und Vertreter (Nürnberg 1890)

Steinke, Hubert, Der nützliche Brief. Die Korrespondenz zwischen Albrecht von Haller und Christoph Jakob Trew 1733-1763, Studia Halleriana, 5 (Basel 1999) 
Stolberg, Michael, ««Mein äskulapisches Orakel!〉 Patientenbriefe als Quelle einer Kulturgeschichte der Krankheitserfahrung im 18. Jahrhundert», Österreichische Zeitschrift für Geschichte 7 (1996) 385-404

- «Krankheitserfahrung und Arzt-Patienten-Beziehung in Samuel Hahnemanns Patientenkorrespondenz», Medizin, Gesellschaft und Geschichte 18 (1999) 169-188

- Homo patiens. Krankheitserfahrung in der Frühen Neuzeit (Köln 2003)

Stukenbrock, Karin, «Der zerstückte Cörper». Zur Sozialgeschichte der anatomischen Sektionen in der frühen Neuzeit (1650-1800), Medizin, Gesellschaft und Geschichte, Beihefte, 16 (Stuttgart 2001)

Taylor, Therese, “'Purgatory on Earth'. An Account of Breast Cancer from Nineteenth-Century France", Social History of Medicine 11 (1998) 381-402

Trew, Christoph Jacob, Gründliche Nachricht dessen was bey einer raren Haupt-Wunden [...] sowohl währender [!] Cur als auch erfolgter Section observirt worden (Nürnberg 1724)

- Vertheidigung der Anatomie (Nürnberg 1729)

- «Observationes botanicae circa Pseudorchidem et Dracunculum palustrem, sive radice arundinacea Plinii, C. B. Pin.», Commercium Litterarium 1 (1731) 60-62

- Osteologie oder eigentliche Fürstellung und Beschreibung aller Beine eines erwachsenen Menschlichen Cörpers (Nürnberg 1740)

- Hortus nitidissimus, omnem per annum superbiens floribus, sive amoenissimarum florum imagines, 3 Bde. (Nürnberg 1750-1786)

- Plantae rariores. Decas I-III (Nürnberg 1763-1784)

Triebs, Michaela, Die Medizinische Fakultät der Universität Helmstedt (1576-1810). Eine Studie zu ihrer Geschichte unter besonderer Berücksichtigung der Promotions- und Übungsdisputationen, Repertorien zur Erforschung der frühen Neuzeit, 14 (Wiesbaden 1995)

Universität Helmstedt (Hrsg.), Monumentorum Sylloge Quibus Memoriam [...] Io. Friderici Crellii (Helmstedt 1747)

Wegner, Richard N., «Christoph Jacob Trew (1695-1769). Ein Führer zur Blütezeit naturwissenschaftlicher Abbildungswerke in Nürnberg im 18. Jahrhundert», Mitteilungen zur Geschichte der Medizin, der Naturwissenschaften und der Technik 39 (1940) 218-228

Wild, Wayne, Medicine-by-post in eighteenth-century Britain. The changing rhetoric of illness in doctor-patient correspondence and literature. Maschinenschrift (Diss. phil. Brandeis University 2001) 


\section{Übersicht des Briefwechsels zwischen Lorenz Heister und Christoph Jacob Trew}

Die Schreiben sind im folgenden chronologisch aufgelistet. Die Verzeichnung mit dem Nachnamen des Autors folgt der Systematik bei Schmidt-Herrling 1940. Bei den Briefen Heisters handelt es sich, soweit nicht anders ausgewiesen, stets um die an Trew gesandten Originalschreiben.

\begin{tabular}{|c|c|c|c|}
\hline Heister & 45 & 9.2 .1717 & \\
\hline Heister & 46 & 10.12.1717 & \\
\hline Heister & 47 & Anfang 1718 & \\
\hline Heister & 48 & 5.3 .1720 & \\
\hline Heister & 49 & 12.5 .1730 & \\
\hline Heister & 50 & 30.6 .1730 & \\
\hline Trew & 321 & 1.7.1730 & $\begin{array}{l}\text { Original; dazu: «Libri excerpti ex Catalogo } \\
\text { Bibliothecae Schmidianae» }(2 \mathrm{~S} .)\end{array}$ \\
\hline Heister & 51 & 4.8.1730 & $\begin{array}{l}\text { nebst handschriftlichem Verzeichnis «Libri } \\
\text { ex catalogo Bibliothecae Schmidianae» (4 S.) }\end{array}$ \\
\hline Heister & 52 & 12.9.1730 & \\
\hline Heister & 53 & 17.10.1730 & \\
\hline Heister & 54 & 1.12 .1730 & \\
\hline Heister & 55 & 29.12 .1730 & \\
\hline Trew & 322 & o. D. (1731?) & Entwurf \\
\hline Trew & 323 & 23.2.1731 & Original \\
\hline Trew & 324 & 23.2.1731 & Entwurf zu Trew 323 \\
\hline Heister & 56 & 28.3.1731 & \\
\hline Heister & 57 & 11.5.1731 & \\
\hline Heister & 58 & 21.8.1731 & \\
\hline Heister & 59 & 3.12 .1731 & \\
\hline Trew & 325 & 13.1.1732 & Original \\
\hline Heister & 60 & 11.2.1732 & \\
\hline Trew & 326 & 26.4.1732 & Original \\
\hline Trew & 327 & 5.10 .1732 & $\begin{array}{l}\text { Entwurf; dazu handschriftliches Pflanzen- } \\
\text { verzeichnis (1 S.) }\end{array}$ \\
\hline Heister & 61 & 5.1 .1733 & \\
\hline Heister & 62 & 8.2.1733 & $\begin{array}{l}\text { Elias Friedrich Heister im Auftrag seines } \\
\text { Vaters }\end{array}$ \\
\hline Heister & 63 & 15.5.1733 & \\
\hline Heister & 64 & 9.11 .1733 & \\
\hline Heister & 65 & 19.4.1734 & \\
\hline Trew & 328 & 1.5.1734 & Entwurf \\
\hline Heister & 66 & 12.7.1734 & \\
\hline Heister & 67 & 30.8 .1734 & \\
\hline Heister & 68 & 4.3.1735 & \\
\hline Heister & 69 & 30.1 .1736 & $\begin{array}{l}\text { nebst handschriftlichem Verzeichnis «Semina } \\
\text { sive Plantae, quae prae ceteris desiderantur } \\
\text { a Laurentio Heistero» } 4 \mathrm{~S} \text {.) }\end{array}$ \\
\hline
\end{tabular}




\begin{tabular}{|c|c|c|c|}
\hline Trew & 329 & 2.4 .1742 & Original \\
\hline Trew & 330 & 2.4.1742 & Entwurf zu Trew 329 \\
\hline Trew & 331 & 15.9.1742 & Entwurf \\
\hline Heister & 70 & 25.3 .1743 & \\
\hline Heister & 71 & 28.6.1743 & \\
\hline Trew & 332 & 9.5.1744 & Entwurf \\
\hline Heister & 72 & 8.4.1745 & \\
\hline Heister & 73 & 31.3.1746 & $\begin{array}{l}\text { nebst gedrucktem Verzeichnis «Semina } \\
\text { et plantae, quae desiderantur pro horto } \\
\text { Helmstadiensi }[\ldots] »(2 \mathrm{~S} .)\end{array}$ \\
\hline Heister & Beilage & o. D. & Verzeichnis, identisch mit Heister 73 \\
\hline Heister & 74 & 18.4.1747 & \\
\hline Trew & 333 & 2.8 .1747 & Entwurf \\
\hline Heister & 75 & 11.8.1747 & \\
\hline Heister & 76 & 17.9.1747 & \\
\hline Heister & 100 & 20.11.1747 & $\begin{array}{l}\text { «Extract Schreibens von Herrn Professor } \\
\text { Heister aus Helmstädt [...]», } \\
\text { von ungenanntem Schreiber }\end{array}$ \\
\hline Heister & 77 & 21.11.1747 & \\
\hline Heister & 78 & 19.12.1747 & \\
\hline Heister & 79 & 16.2.1748 & \\
\hline Heister & 80 & 26.3.1748 & \\
\hline Heister & 81 & 23.4.1748 & \\
\hline Heister & 82 & 21.5.1748 & \\
\hline Heister & 83 & 16.7.1748 & \\
\hline Heister & 84 & 20.8 .1748 & \\
\hline Heister & 85 & nach 19.9.1748 & \\
\hline Heister & 86 & 4.3 .1749 & $\begin{array}{l}\text { von anderer Hand geschriebene } \\
\text { Traueranzeige für Eva Maria Heister } \\
\text { mit Ergänzungen von } \\
\text { Lorenz Heister }\end{array}$ \\
\hline Heister & 87 & 19.11.1748 & \\
\hline Trew & 334 & 3.2 .1750 & Entwurf \\
\hline Heister & 101 & vor 21.7.1750 & $\begin{array}{l}\text { «Aus Herrn Hofrath Heisters Schreiben }[\ldots] » \text {, } \\
\text { von ungenanntem Schreiber }\end{array}$ \\
\hline Heister & 88 & nach 9.10.1750 & \\
\hline Heister & 89 & 3.11 .1750 & \\
\hline Heister & 90 & 29.1.1751 & \\
\hline Heister & 91 & 26.3.1751 & \\
\hline Heister & 92 & 21.5.1751 & \\
\hline Heister & 93 & 30.9.1751 & \\
\hline Heister & 94 & 8.10 .1751 & \\
\hline Heister & 95 & 6.4 .1753 & \\
\hline Heister & 102 & 15.6.1753 & $\begin{array}{l}\text { «xtract Schreibens von Herrn Hofrath } \\
\text { Heister }[\ldots . .] » \\
\text { von ungenanntem Schreiber }\end{array}$ \\
\hline
\end{tabular}




$\begin{array}{lrrl}\text { Trew } & 335 & 6.6 .1755 & \begin{array}{l}\text { beinhaltet Trews «Historia morbi des von } \\ \text { Holzschuer» }(8 \mathrm{~S} \text {.) in Original und Abschrift } \\ \text { sowie Heisters Entwurf für sein «Consilium } \\ \text { medicum» }\end{array} \\ \text { Heister } & 96 & 14.5 .1756 & \begin{array}{l}\text { gedruckte Traueranzeige für Catharina Maria } \\ \text { Dorothea Heister, verwitwete Harding } \\ \text { Original }\end{array} \\ \text { Trew } & 336 & 7.6 .1756 & \begin{array}{l}\text { Entwurf zu Trew 336 } \\ \text { Trew }\end{array} \\ \text { Heister } & 937 & 2.8 .1756 & \\ \text { Trew } & 338 & 16.11 .1756 & \text { Entwurf } \\ \text { Heister } & 98 & 7.1 .1757 & \text { mit beigelegtem Kupferstich } \\ \text { Heister } & 99 & 20.12 .1757 & \\ & & & \end{array}$

